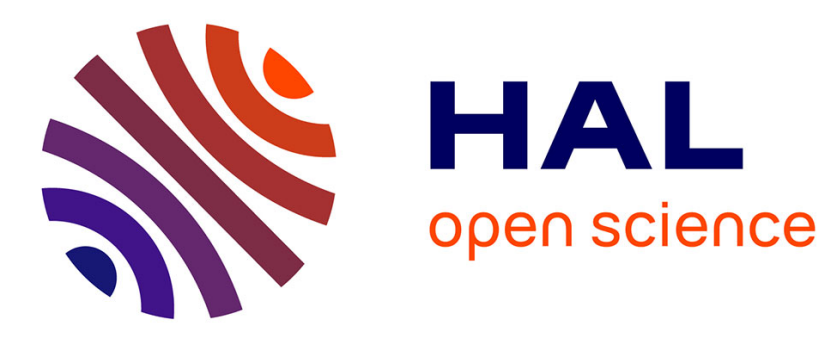

\title{
Cycles in digraphs- a survey
}

Jean-Claude Bermond, Carsten Thomassen

\section{To cite this version:}

Jean-Claude Bermond, Carsten Thomassen. Cycles in digraphs- a survey. Journal of Graph Theory, 1981, 5 (1), pp.1-43. 10.1002/jgt.3190050102 . hal-02424283

\section{HAL Id: hal-02424283 \\ https://hal.inria.fr/hal-02424283}

Submitted on 26 Dec 2019

HAL is a multi-disciplinary open access archive for the deposit and dissemination of scientific research documents, whether they are published or not. The documents may come from teaching and research institutions in France or abroad, or from public or private research centers.
L'archive ouverte pluridisciplinaire HAL, est destinée au dépôt et à la diffusion de documents scientifiques de niveau recherche, publiés ou non, émanant des établissements d'enseignement et de recherche français ou étrangers, des laboratoires publics ou privés. 


\title{
Cycles in Digraphs- A Survey
}

J.C. Bermond

UNIVERSITÉ PARIS-SUD

ORSAY, FRANCE

C. Thomassen

MATEMATISK INSTITUT, AARHUS UNIVERSITET

AARHUS, DENMARK

\begin{abstract}
The main subjects of this survey paper are Hamiltonian cycles, cycles of prescribed lengths, cycles in tournaments, and partitions, packings, and coverings by cycles. Several unsolved problems and a bibliography are included.
\end{abstract}

\section{INTRODUCTION AND TERMINOLOGY}

The concept of a cycle plays a fundamental role in the theory of undirected graphs, and there are numerous papers dealing with cycles. The literature on cycles in digraphs is not so extensive. This is partly because cycles do not play the same fundamental role for directed graphs as for undirected graphs. For example, the cycles in a digraph are not, in general, the circuits of a 1 matroid, as is the case for undirected graphs, and if one considers the digraph of an electrical network, it is the cycles of the underlying undirected graph (rather than those of the digraph) that are of primary interest. However, the main reason for the relatively few results on cycles in digraphs is probably that it is considerably more difficult to study these. The cycles in an undirected graph correspond in an obvious way to the cycles of length three or more in the symmetric digraph associated with the graph; so it often happens that a result on cycles in undirected graphs has a natural, but more difficult, generalization to digraphs.

The purpose of this paper is to survey results on cycles in digraphs and compare them with the analogous results for undirected graphs, and also to present a list of unsolved problems on the subject. We treat Hamiltonian cycles (Sec. 1), cycles of prescribed lengths (Sec. 2), cycles in tournaments (Sec. 3), and coverings and packings with cycles (Sec. 4), and we mention problems of a more special nature (Sec. 5). 
For the problem of counting cycles in digraphs the reader is referred to Harary and Palmer (1973). The related problem of listing all cycles in a digraph has received much attention because of its importance in optimizing computer programs. Algorithms for this problem having $0(n+e+e c)$ time requirements (where $n, e, c$ are the numbers of vertices, arcs, and distinct cycles, respectively, in the digraph under consideration) have been described by Johnson (1975), Read and Tarjan (1975), and Szwarcfiter and Lauer (1974). These and other algorithms are discussed in Mateti and Deo (1976).

Also, the Traveling Salesman Problem, i.e., the problem of finding a Hamiltonian cycle of minimum weight in a digraph with weighted arcs, has many applications and has been treated in several papers. For a survey on this problem, the reader is referred to Bellmore and Nemhauser (1968) and Christofides (1980), and a bibliography is given by Pierce (1975).

For cycles in undirected graphs, the reader is referred to the monograph of Walther and Voss (1974) and the survey articles by Bermond (1979), Bondy (1978), and Lesniak-Foster (1977). Some of the results on cycles in tournaments are treated in more detail in the monograph of Moon (1968) and in the survey paper of Beineke and Reid (1979), and long cycles in digraphs are also treated in the survey paper of Thomassen (1979).

We use standard terminology. For the sake of clarity we repeat the most important definitions:

A digraph (directed graph) $\mathbb{D}$ is a pair $(\mathbb{X}, U)$, where $X$ is a finite set of elements, called vertices, and $U$ is a set of ordered pairs $(x, y)$ of vertices, called arcs. If the $\operatorname{arc}(x, y)$ is present, we say that $x$ dominates $y$. The number of vertices of $\mathbb{D}$ is the order of $\mathbb{D}$.

A path is a digraph $(\mathbb{X}, \mathbb{U})$, where $X=\left\{x_{1}, x_{2}, \ldots, x_{n}\right\}$ and $U=\left\{\left(x_{i}, x_{i+1}\right) \mid \mathbb{1}\right.$ $\leq i \leq n-1\}$. If we add the arc $\left(x_{n}, x_{1}\right)$, we obtain a cycle (in this case a cycle of length $n$ or an $n$-cycle). The cycle of length $n$ is denoted by $\mathbf{C}_{n}$.

A cycle in a digraph $D$ including all vertices of $D$ is a Hamiltonian cycle and, if $D$ has such a cycle, we say that $\mathbb{D}$ is Hamiltonian.

An oriented graph is a digraph with no 2-cycle, and a tournament is an oriented graph in which any two vertices are adjacent.

A digraph is symmetric if every arc is contained in a 2-cycle. If $G$ is an undirected graph, we denote by $G^{*}$ the symmetric digraph associated with $G$. The converse digraph of $D$ is the digraph obtained from $D$ by reversing the directions of all arcs.

A digraph $\mathbb{D}$ is strong, if for any two vertices $x$ and $y, D$ contains a path from $x$ to $y$ and a path from $y$ to $x . D$ is $k$-connected if the deletion of fewer than $k$ vertices always results in a strong digraph. A $k$-arc-connected digraph is defined analogously. A component of a digraph $D$ is a maximal strong subdigraph. The components of $\mathbb{D}$ can be labeled $D_{1}, D_{2}, \ldots, D_{k}$ such that no vertex of $D_{i}$ dominates a vertex of $D_{j}$ if $j<i$. If $D$ is a tournament, this 
labelling is unique, and we refer to $D_{1}$ and $D_{k}$ as the initial, respectively terminal, components of $D$, and the other components are intermediate components.

The outdegree $d^{+}(x)$ of a vertex $x$ in $D$ is the number of arcs starting at $x$ and the indegree $d^{-}(x)$ is the number of arcs terminating at $x$. The total degree (or just degree) of $x$ is defined by $d(x)=d^{+}(x)+d^{-}(x)$.

$D$ is $k$-diregular if $d^{+}(x)=d^{-}(x)=k$ for each vertex, and $D$ is $k$-regular if $d(x)=k$ for each vertex $x$.

The cartesian product $D_{1} \times D_{2}$ of two digraphs $D_{1}=\left(X_{1}, U_{1}\right)$ and $D_{2}=\left(X_{2}, U_{2}\right)$ is the digraph with vertex set $X_{1} \times X_{2}$ such that a vertex $\left(x_{1}, x_{2}\right)$ dominates $\left(y_{1}, y_{2}\right)$ if and only if $x_{1}=y_{1}$ and $\left(x_{2}, y_{2}\right) \in U_{2}$, or $x_{2}=y_{2}$ and $\left(x_{1}, y_{1}\right) \in U_{1}$.

The lexicographic product $\mathbb{D}_{1} \otimes \mathbb{D}_{2}$ is the digraph with vertex set $X_{1} \times X_{2}$ such that $\left(x_{1}, x_{2}\right)$ dominates $\left(y_{1}, y_{2}\right)$ if and only if $x_{1}$ dominates $y_{1}$, or $x_{1}=y_{1}$ and $x_{2}$ dominates $y_{2}$.

In the figures two oppositely oriented arcs joining the same vertices will be represented by an undirected edge.

For any real number $x,|x|$ denotes the integer part of $x$, and $[x|=-|-x\rfloor$.

\section{HAMILTONIAN CYCLES}

\subsection{Sufficient Conditions on the Degrees}

Two fundamental early results on Hamiltonian cycles in digraphs are those of Camion and Ghouila-Houri.

Theorem 1.1.1 [Camion (1959)]. A tournament is Hamiltonian if and only if it is strong.

Theorem 1.1.2 [Ghouila-Houri (1960)]. Every strong digraph of order $n$ and minimum degree at least $n$ is Hamiltonian.

Ghouila-Houri's theorem implies the well known theorem of Dirac (1952) that every undirected graph of order $n$ and minimum degree at least $n / 2$ is Hamiltonian. Ore (1960) proved that the same conclusion holds if we only assume that the sum of the degrees of any two nonadjacent vertices is at least $n$. This was generalized to digraphs by Woodall.

Theorem 1.1.3 [Woodall (1972)]. A digraph $D$ of order $n$ is Hamiltonian if, for any two vertices $x$ and $y$, either $x$ dominates $y$ or

$$
d^{+}(x)+d^{-}(y) \geq n .
$$


A common generalization of Theorems 1.1.1,1.1.2, and 1.1.3 was obtained by Meyniel.

Theorem 1.1.4 [Meyniel (1973)]. A strong digraph $D$ of order $n$ is Hamiltonian if for any two nonadjacent vertices $x$ and $y$ we have

$$
d(x)+d(y) \geq 2 n-1 .
$$

Meyniel's original proof is lengthy, but a short proof was found by OverbeckLarish (1976) and a slightly simpler proof was given by Bondy and Thomassen (1977).

The proof of Bondy and Thomassen is constructive and yields an efficient algorithm in $0\left(n^{4}\right)$ steps for finding a Hamiltonian cycle in a digraph satisfying the hypothesis of Meyniel's theorem [see Minoux (1980) or Bermond (1979)].

Theorems 1.1.2, 1.1.3, and 1.1.4 are best possible in the sense that they become false if the degree conditions are relaxed. This can be demonstrated by the complete bipartite digraphs with the property that the difference between the cardinalities of the color-classes is one. Another example is given by the following digraph $\mathbb{H}_{n}$. Let $u$ be a vertex of $\mathbb{K}_{n-2}^{*}$, the complete symmetric digraph of order $n-2$. Obtain digraph $\mathbb{H}_{n}$ by adding two new vertices $v$ and $w$, each of which dominates all $n-2$ vertices of $K_{n-2}^{*}$ and is dominated by only $u$.

Clearly $H_{n}$ is non- $\mathbb{H}$ amiltonian; however, the degree of $u$ is $2 n-2$, and the degree of any other vertex different from $v$ and $w$ is $2 n-4$; only $v$ and $w$ have degree $n-1$. It is an immediate consequence of Meyniel's theorem that $\mathbb{H}_{n}$ contains the maximum number of arcs possible for a strong non-Hamiltonian digraph. A stronger assertion is given in Theorem 1.3.1 below.

The requirement in Theorems 1.1.2 and 1.1.4 that $D$ be strong is necessary as is demonstrated by a digraph consisting of two complete symmetric digraphs joined completely by arcs all in the same direction.

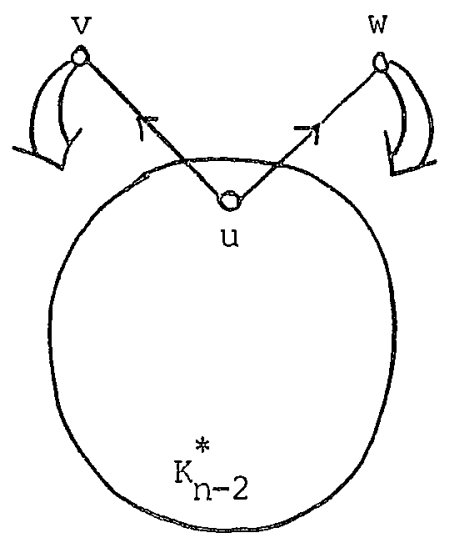

FIGURE 1. The digraph $H_{n}$. 
Häggkvist (1977) has used Meyniel's theorem to obtain results on the existence of Hamiltonian paths and cycles containing prescribing edges in an undirected graph. For example, if the sum of the degrees of any two nonadjacent vertices $x$ and $y$ in an undirected graph with $n$ vertices, where $n$ is even, is at least $n+1$ (resp. $n-1$ ), then any 1 -factor of the graph can be extended into a Hamiltonian cycle (resp. path). This result is best possible in the sense that $n+1$ (resp. $n-1$ ) cannot be replaced by $n$ (resp. $n-2)$. An analogous result for bipartite graphs was obtained by Las Vergnas (1971).

\subsection{Conjectures on the Degree Sequence}

Pósa (1962) generalized Ore's theorem by showing than an undirected graph has a Hamiltonian cycle provided its degree sequence majorizes the sequence $2,3, \ldots,[(n-1) / 2],[n / 2],[(n+1) / 2], \ldots,[(n+1) / 2]$. NashWilliams $(1968,1969)$ proposed a generalization of this to digraphs. Specifically, he made the following conjecture:

Conjecture 1.2.1 [Nash-Williams $(1968,1969)]$. If both the sequence of outdegrees and the sequence of indegrees of a digraph $D$ of order $n$ majorize the sequence $2,3, \ldots,[(n-1) / 2],[n / 2],[(n+1) / 2], \ldots,[(n+1) / 2]$, then $D$ has a Hamiltonian cycle.

This seems to be a difficult conjecture. As noted by Nash-Williams (1969), it is not even obvious that in a digraph satisfying the assumption of the conjecture, there is a cycle through any two vertices.

One may also try to obtain digraph analogues of various other sufficient conditions on the degrees, such as Chvátal's theorem (1972) which asserts that if the degree sequence $d_{1} \leq d_{2} \cdots \leq d_{n}$ of an undirected graph satisfies the condition $d_{k} \leq k<n / 2 \Rightarrow d_{n-k} \geq n-k$, then the graph is Hamiltonian. In particular, we may ask whether every strong digraph whose nondecreasing degree sequence $d_{1} \leq d_{2} \cdots \leq d_{n}$ satisfies the following condition is Hamiltonian:

$$
d_{k} \leq 2 k<n \Rightarrow d_{n-k} \geq 2(n-k) \quad \text { for each } k .
$$

Similarly one may ask whether every strong digraph whose nondecreasing outdegree and indegree sequences $d_{1}^{+} \leq d_{2}^{+} \cdots \leq d_{n}^{+}$and $d_{1}^{-} \leq d_{2}^{-} \cdots \leq d_{n}^{-}$ satisfy the following conditions is Hamiltonian:

$$
\begin{aligned}
& d_{k}^{+} \leq k<\frac{n}{2} \Rightarrow d_{n-k}^{+} \geq n-k \quad \text { and } \\
& \qquad d_{k}^{-} \leq k<\frac{n}{2} \Rightarrow d_{n-k}^{-} \geq n-k .
\end{aligned}
$$

In fact, the digraph $\mathbb{H}_{n}$ of Figure $\mathbb{1}$ shows that the answer to each of these questions is negative. A third possible analogue of Chvátal's theorem, which survives $H_{n}$ is the following conjecture due to Nash-Williams (1975). 
Conjecture 1.2.2 [Nash-Williams (1975)]. If $D$ is a strong digraph whose nondecreasing indegree and outdegree sequences $d_{1}^{+} \leq d_{2}^{+} \cdots \leq d_{n}^{+}$and $d_{1}^{-} \leq d_{2}^{-} \cdots \leq d_{n}^{-}$satisfy the conditions:

$$
d_{k}^{+} \leq k<\frac{n}{2} \Rightarrow d_{n-k}^{-} \geq n-k \text { and } d_{k}^{-} \leq k<\frac{n}{2} \Rightarrow d_{n-k}^{+} \geq n-k
$$

for each $k$, then $D$ is Hamiltonian.

\subsection{Conditions on the number of arcs}

Ore (1961) proved that, if an undirected graph $G$ of order $n$ has more than $\left(\begin{array}{c}n-1 \\ 2\end{array}\right)+1$ edges, then $G$ is Hamiltonian, and Bondy (1972) showed that the only non-Hamiltonian graphs with $\left(\begin{array}{c}n-1 \\ 2\end{array}\right)+1$ edges are the graphs $G(1, n)$ consisting of a complete graph $\mathbb{K}_{n-1}$ plus a vertex joined to a given vertex of this $K_{n-1}$ and an exceptional graph of order 5.

These results have been extended to digraphs as follows:

Theorem 1.3.1 [Bermond, Germa, Heydemann, and Sotteau (1980)]. The only non-Hamiltonian strong digraphs with at least $(n-1)(n-2)+2$ arcs are the symmetric digrapln $G(1, n)^{\text {* }}$ the digraph $\mathbb{H}_{n}$ of Figure $\mathbb{1}$ and its converse and the digraphs of figure 2 .

If we do not require $G$ to be strong, we have the following theorem.

Theorem 1.3.2 [Lewin (1975)]. If $D$ is a digraph of order $n$ with more than $(n-1)^{2}$ arcs, then $D$ is Hamiltonian.

This result is best possible in view of the digraph consisting of $\mathbb{K}_{n-1}^{*}$ plus a new vertex which dominates or is dominated by all the vertices of the $\mathbb{K}_{n-1}^{*}$.

To conclude this section we mention a problem of a probabilistic nature posed independently by Wright (1973) and Bondy (1978):
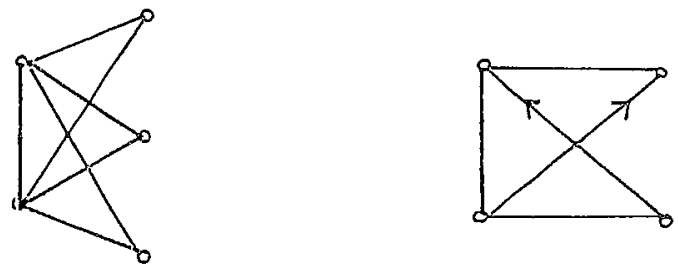

FIGURE 2. Non-Hamiltonian digraphs with largest possible number of arcs. 
Problem 1.3.3. Determine the asymtotically smallest function $f(n)$ such that a digraph of order $n$ with $f(n)$ arcs randomly placed is almost certainly Hamiltonian (that is, the probability that the resulting digraph is Hamiltonian tends to 1 as $n$ tends to infinity).

In the undirected case this function has been shown to be $\frac{1}{2} n \log n+\frac{1}{2} n$ $\log \log n+O(n)$ by Komlós and Szemerédi (1976) and Koršunov (1976) improving an earlier result of Pósa (1976). Cycles in random digraphs have been investigated by Palesti (1971).

\subsection{Strong Digraphs with Minimum Degree $m-1$ and Regular Digraphs}

As another possible generalization of Ghouila-Houri's theorem, NashWilliams (1969) suggested the problem of characterizing the strong digraphs of order $n$ and minimum degree $n-1$ that have no Hamiltonian cycle. A result of this kind for undirected graphs can be obtained from work of Dirac (1973): the only non-Hamiltonian undirected graphs of order $n$ ( $n$ odd) and minimum degree $(n-1) / 2$ are the graph obtained from two complete graphs each with $(n+1) / 2$ vertices by identifying a vertex in one with a vertex in the other, and the graphs obtained from the complete bipartite graph $K_{(n-1) / 2,(n+1) / 2}$, by adding some edges joining vertices of degree more than $\frac{1}{2}(n-1)$. The symmetric digraphs associated with these graphs are nonHamiltonian and have minimum degree $n-1$, but they are not the only ones. Figure 3 shows two other examples. Because none of the undirected graphs above are regular, it follows that every $((n-1) / 2)$-regular graph with $n$ vertices $(n$ odd) is Hamiltonian, a result first proved by Nash-Williams (1970).

Bondy (1978) proposed the following generalization of the above to digraphs: Every $((n-1) / 2)$-diregular digraph $\mathbb{D}$ of order $n$ ( $n$ odd) is Hamiltonian or is isomorphic to $D_{5}$ or $D_{7}$ (Fig. 3 ). This conjecture was
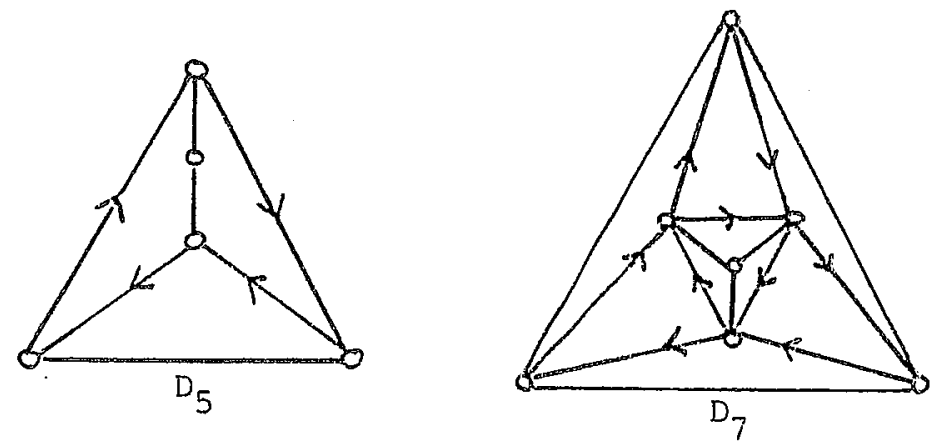

FIGURE 3. Two non-Hamiltonian diregular digraphs. 
proved by Thomassen (1980) (see Theorem 1.4.4). Bondy also made the stronger conjecture that every strong $(n-1)$-regular digraph is Hamiltonian (except $D_{5}$ and $D_{7}$ ). This conjecture turned out to be false. Thomassen (1980) described a variety of counterexamples. Consider, for example, the digraph obtained from two disjoint complete symmetric digraphs $D_{1}$ and $D_{2}$ by identifying a vertex of $D_{1}$ with a vertex of $D_{2}$. Then add two new vertices $u$ and $v$ and let each vertex of $D_{1}$ (resp. $D_{2}$ ) dominate (resp. be dominated by) each vertex not in $D_{1}$ (resp. $D_{2}$ ). It is easy to find a spanning strong $(n-1)$ regular subdigraph of this digraph. These examples and other examples in Thomassen (1980) indicate that it is not easy to describe the strong nonHamiltonian digraphs of order $n$ and minimum degree $n-1$. All the examples that we know of have connectivity one, so the following conjecture may be true:

Conjecture 1.4.1 [Thomassen (1979)]. Every 2-connected $(n-1)$-regular digraph of order $n$, except $D_{5}$ and $D_{7}$, is Hamiltonian.

As a partial solution to the above problem of Nash-Williams, Thomassen (1980), proved the following:

Theorem 1.4.2 [Thomassen (1981)]. If $D$ is a strong digraph of order $n$ and minimum degree $n-1$, and if $S$ is any longest cycle of $D$, then every vertex of $D-V(S)$ has degree $n-1$, any two vertices of $D-V(S)$ are adjacent, and every component of $D-V(S)$ is a complete digraph. Moreover, if $\mathbb{D}$ is 2-connected, then $S$ can be chosen such that $\mathbb{D}-V(S)$ is a transitive tournament.

Additionally, Thomassen (1981) showed that a component of $\mathbb{D}-\mathbb{V}(\mathbb{S})$ may have any order $s, s<n / 2$.

We have already described many strong non-Hamiltonian digraphs of order $n=2 k+1$ and minimum degree $n-1$. If we impose the additional condition that all indegrees and outdegrees be at least $k$, the class of digraphs obtained is considerably smaller.

Theorem 1.4.3 [Thomassen (1981)]. Let $D$ be a digraph of order $n=2 k+1$ and minimum indegree and outdegree at least $k$. Then $D$ is HIamiltonian unless $D$ has a set of $k+1$ mutually nonadjacent vertices (which then dominate and are dominated by all the $k$ remaining vertices), or $D$ is isomorphic to $D_{5}$ or $D_{7}$ of Figure 3 , or $D$ is the symmetric digraph consisting of two disjoint copies of $K_{k}^{*}$ plus one vertex joined to all others by two arcs.

Since $D_{5}$ and $D_{7}$ are the only diregular digraphs in Theorem 1.4.3, the above-mentioned conjecture of Bondy on Hamiltonian cycles in diregular digraphs follows.

Theorem 1.4.4 [Thomassen (1981)]. If $D$ is a $k$-diregular digraph of order $n=2 k+1$, the $D$ is Hamiltonian unless $\mathbb{D}$ is isomorphic to $\mathbb{D}_{5}$ or $\mathbb{D}_{7}$ of Figure 3 . 
Jackson (1980) proved that every 2-connected undirected $k$-regular graph with at most $3 k$ vertices is Hamiltonian. This cannot be extended to digraphs. Thomassen (1981) proved that, for each $k$, there are infinitely many $k$ diregular digraphs with no cycle of length $>k+3$. To see this, we consider $m$ disjoint copies $D_{1}, D_{2}, \ldots, D_{m}$ of $K_{k+1}^{*}$. For each $i, 1 \leq i \leq m-1$, select vertices $x_{i}, y_{i}$ in $D_{i}$ and vertices $z_{i+1}, v_{i+1}$ in $D_{i+1}$, delete the $\operatorname{arcs}\left(x_{i}, y_{i}\right)$, $\left(y_{i}, x_{i}\right),\left(z_{i+1}, v_{i+1}\right),\left(v_{i+1}, z_{i+1}\right)$ and add the 4-cycle $\left(x_{i}, z_{i+1}, y_{i}, v_{i+1}, x_{i}\right)$. Then a longest cycle in the resulting digraph has length $k+3$ if $m \geq 3$ and $k+2$ if $m=2$.

We return to diregular digraphs in Sec. 4, which deals with Hamiltonian decompositions. Here we mention two conjectures concerning diregular digraphs.

Conjecture 1.4.5 [Jackson (1980b)]. If $k \geq 3$, then every $k$-diregular oriented graph with at most $4 k+1$ vertices is Hamiltonian.

Jackson (1980b) described a 2-diregular non-Hamiltonian oriented graph of order 8 and, by modifying the construction given above (with $m=2$ ), one can construct, for each $k \geq 2$, a $k$-diregular non-Hamiltonian oriented graph of order $4 k+4$.

Conjecture 1.4.6 [Thomassen (1979)]. Every oriented graph of order $n$ in which each indegree and outdegree is at least $n / 3$ is Hamiltonian.*

The oriented graph with vertex set $A \cup \mathbb{B} \cup \mathbb{C}$ and arc set $\{(x, y) \mid x \in$ $A, y \in \mathbb{B}$, or $x \in \mathbb{B}, y \in C$, or $x \in C, y \in A\}$ is HIamiltonian if and only if $|A|=|B|=|C|$. So Conjecture 1.4.6 is best possible.

The first nontrivial step towards a proof of Conjecture 1.4.6 was made by Jackson (1981).

Theorem 1.4.7 [Jackson (1981)]. Let $D$ be an oriented graph with minimum indegree and outdegree at least $k(k \geq 2)$ and with at most $2 k+2$ vertices. Then $D$ is Hamiltonian.

Jackson (1981) also proved that an oriented grtaph $D$ in which each indegree and outdegree is at least $k$ contains a path of length at least $2 k$. He made the conjecture that $D$ even contains a path of length at least $3 k$ (or a Hamiltonian path) if it is strong.*

For large digraphs the following result is a drastic extension of Theorem 1.4.7.

\footnotetext{
*R. Häggkvist (private communication) has disproved Conjecture 1.4.6 and his counterexamples can be modified to disprove the $3 k$-path-conjecture of Jackson as well.
} 
Theorem 1.4.8 [Thomassen (1980c)]. There exists a positive constant $c$ such that an oriented graph $D$ of order $n$ is Hamiltonian provided each vertex has indegree and outdegree at least $n / 2-c \sqrt{n}$.

\subsection{Other Sufficient Conditions for Hamiltonian Cycles}

In this section we comment on connectivity, independence number, powers of digraphs, planar digraphs, and line digraphs.

The Chvátal-Erdös Theorem (1972) asserts that an undirected graph $G$ is Hamiltonian provided that its connectivity is not less than its independence number (or stability number). For digraphs each of the following three invariants is the independence number, when restricted to undirected graphs. If $D$ is a digraph, then $i_{1}(D)$ (resp. $i_{2}(D)$, resp. $i_{3}(D)$ ) is the maximum cardinality of a vertex set $A$ of $\mathbb{D}$ such that $D(A)$ has no arc (resp. no cycle, resp. no 2-cycle).

The invariant $i_{2}(D)$ was studied by Meyniel (1980). Las Vergnas and Meyniel (Seminar, Paris 1977) and Bondy (1978) conjectured that a $k$ connected digraph $D$ with $i_{2}(D) \leq k$ is Hamiltonian or isomorphic to $\mathbb{D}_{7}$ of Figure 3. However, Thomassen (1980) has exhibited infinite families of counterexamples to the case $k=3$ of this conjecture. Mioreover, the construction preceding Conjecture 1.4 .5 (with $m=2$ ) can easily be modified to given non-Hamiltonian 2-connected digraphs with $i_{3}=2$.

Las Vergnas (private communication) has introduced the following stronger connectivity concept for digraphs. A digraph is $(k, l)$-connected if, for every pair of vertices $x$ and $y$, there exist $k$ paths from $x$ to $y$ and $\ell$ paths from $y$ to $x$, all the paths having only $x$ and $y$ in common. Las Vergnas (private communication) conjectured that a digraph $\mathbb{D}$ is Hamiltonian provided $i_{2}(D) \leq k$ and $\mathbb{D}$ is $(h, k-h)$-connected for each $h, 0 \leq h \leq k$.

However, it is no easier to work with this connectivity concept than it is to decide whether or not a digraph is Hamiltonian. Indeed, Fortune, Hopcroft, and Wyllie (1980) have proved that the problem of deciding whether two vertices of a digraph are on a common cycle is NP-complete (see also Problem 1.5.2). This problem was mentioned by Frank (1978).

Let $D^{p}$, the pth power of the digraph $D$, be the digraph having the same vertex set as $D$, such that there is an $\operatorname{arc} D^{p}$ from $x$ to $y$ if and only if there exists a path from $x$ to $y$ of length less than or equal to $p$ in $D$.

In the undirected case, Sekanina (1960) proved that the cube of every connected graph is Hamiltonian, and Fouquet (1978) has shown that this has no immediate extension to digraphs. Specifically, he proved the following:

Theorem 1.5.1 [Fouquet (1978)]. For every $p$ and $q$ there exists a $q$ connected digraph $D$ such that $D^{p}$ is not Hamiltonian. 
He proved, however, that if $D$ contains a cycle of length $k$, then $D^{n-k-1}$ is Hamiltonian.

Bermond (1980) conjectured the following possible generalization of Sekanina's result: If $D$ is connected and Eulerian [that is $d^{+}(x)=d^{-}(x)$ for every vertex $x$ ], then $D^{3}$ is Hamiltonian. Pács (private communication) gave infinite families of counterexamples. Let the vertex set of $D$ be the disjoint union of $X_{1}, X_{2}, X_{3}, X_{4}$ with $\left|X_{1}\right|=8 p,\left|X_{2}\right|=4 p,\left|X_{3}\right|=2 p,\left|X_{4}\right|=$ $p \geq 4$. Let the vertices of each $X_{i}, 1 \leq i \leq 4$, be labeled, $1,2, \ldots$. Arcs of $D$ are as follows: From the vertices $2 i-1$ and $2 i(i=1, \ldots, 4 p)$ of $X_{1}$ to the vertex $i$ of $X_{2}$, from the vertices $2 j-1$ and $2 j(j=1 \ldots, 2 p)$ of $X_{2}$ to the vertices $j$ and $j+1$ of $X_{3}$, from the vertices $2 k-1$ and $2 k(k=1, \ldots, p)$ of $X_{3}$ to the vertices $k, k+1, k+2, k+3$ of $X_{4}$ (the indices being taken $\bmod p$ ), and from the vertex $k$ of $X_{4}$ to the vertices $8(k-1)+1, \ldots$, $8(k-1)+8$ of $X_{1}$. Thus, $d^{+}(x)=a^{-}(x)=2^{i-1}$ for $x \in X_{i}$, and so $\mathbb{D}$ is Eulerian. But clearly $\mathbb{D}^{3}$ is not Hamiltonian, since $\mid X_{2} \cup X_{3} \cup$ $X_{4}|<| X_{1} \mid$.

Alpern (1978) gave a sufficient condition, in terms of powers of graphs and digraphs, for a digraph to have a Hamiltonian cycle and applied that to problems concerning measure preserving homeomorphisms in certain measure spaces.

The theorem of Tutte (1956) that every 4-connected planar graph has a Hamiltonian cycle seems to have no natural generalization to digraphs. The maximum connectivity of a planar graph is 5, and a 5-connected nonHamiltonian planar digraph can be obtained as follows. Consider a 5connected undirected planar graph having a face of degree 17 or more (it is not difficult to describe such a graph). Let $x_{1}, x_{2}, \ldots, x_{k}, x_{1}(k \geq 17)$ be the boundary cycle of this face. Replace every edge of the graph by a cycle of length 2 and then add two new vertices $y_{2} z$ and the arcs $\left(y, x_{i}\right)(1 \leq i \leq 5)$, $\left(x_{i}, y\right)(5 \leq i \leq 9),\left(x_{i}, z\right)(9 \leq i \leq 13)$ and $\left(z, x_{i}\right)(13 \leq i \leq 17)$. Then the resulting digraph is planar and 5-connected, but clearly it has no cycle containing both $y$ and $z$.

This example also suggests the following problem:

Problem 1.5.2 [Bermond and Lovász (1975)]. Does there exist a natural number $k$ such that for every $k$-connected digraph $\mathbb{D}$ each pair of vertices belongs to a common cycle? (In view of the above example, $k$ must be at least six.)

Jackson (private communication) conjectured that if $\mathbb{D}$ is oriented, then $k=3$ will suffice.

Problem 1.5.2 is a special case of the following problem: 
Problem 1.5.3. Does there exist, for each pair $k, l$ of natural numbers, a natural number $f(k, l)$ such that every $f(k, l)$-connected digraph is $(k, l)$ connected?

Sometimes it happens, that a problem is easier for digraphs than for graphs. Such is the case with Hamiltonian cycles in line digraphs. Kastelyn (1963) proved that the line digraph of a digraph $D$ is Hamiltonian if and only if $D$ is Eulerian. It is not known when the line graph of an undirected graph $G$ is Hamiltonian. Thomassen conjectures that every 4-connected line graph $L(G)$ is Hamiltonian and he has verified this in the special case when $G$ is 4edge-connected (unpublished).

\subsection{Hamiltonian Problems of a More Special Nature}

The following result of Hamidoune (1979) concerns the length of a Hamiltonian walk (closed walk meeting every vertex at least once) in a digraph with a given connectivity. It disproves a conjecture of Jolivet (1974).

Theorem 1.6.1 [Hamidoune (1979)]. A $k$-connected digraph of order $n$ has a Hamiltonian walk of length at most

$$
\max \left(\left\lfloor\frac{n+k}{2 k}\right\rfloor\left(n-\left\lfloor\frac{n-k}{2 k}\right\rfloor\right),\left\lceil\frac{n-k}{2 k}\right\rceil\left(n-k\left\lceil\frac{n-k}{2 k}\right\rceil\right)\right) .
$$

A very restrictive class of Hamiltonian digraphs was considered by Chartrand, et al. (1969). A digraph is randomly Hamiltonian if every path of the digraph can be extended to a Hamiltonian cycle. They proved the following theorem:

Theorem 1.6.2 [Chartrand, Kronk, and Lick (1969)]. A digraph of order $n$ is randomly Hamiltonian if and only if it is either one of the digraphs $K_{n}^{*}, C_{n}^{*}$ or, for $n$ even, $K_{n / 2, n / 2}^{*}$, or is the lexicographic product of a cycle with an independent set.

The analgous theorem of Chartrand and Kronk (1968) for undirected graphs follows as a special case. It would be interesting to know which digraphs are randomly Hamiltonian from some vertex and which digraphs have the property that every path can be extended into a Hamiltonian path. Such results would generalize Theorem 1.6.2 and the corresponding results for undirected graphs due to Thomassen $(1973,1974)$. Another possible generalization would be to characterize the Hamiltonian digraphs in which every Hamiltonian path can be extended into a Hamiltonian cycle. Dirac and Thomassen (1973) showed that the undirected graphs with this property are 
precisely the randomly Hamiltonian graphs. But for digraphs the situation is different. For example, Grünbaum (1969) showed that there are precisely two tournaments (with three and five vertices, respectively) such that every Hamiltonian path is contained in a Hamiltonian cycle. This result was also obtained by Thomassen (1980a) from some more general results to be mentioned later. The aforementioned problem of characterizing digraphs that are randomly Hamiltonian from some vertex has been solved for tournaments by Thomassen (unpublished) who has proved that for each $n \geq 5$, there are precisely three non-isomorphic tournaments of order $n$ with this property.

Using the abovementioned result of Dirac and Thomassen (1973), Thomassen (1980c) gave a short proof of the following result:

Theorem 1.6.3 [Grötschel and Harary (1979)]. If $G$ is a 2-edge-connected graph, then $G$ has a non-Hamiltonian orientation, unless $G$ is a cycle or a complete graph.

A special class of non-Hamiltonian digraphs are the hypohamiltonian digraphs, i.e., the digraphs $D$ such that $D$ is non-Hamiltonian but every vertex-deleted digraph $D-v$ is Hamiltonian. Undirected hypohamiltonian graphs have been studied to a large extent [for references see, for example, Thomassen (1978)], and the richness of such graphs shows that it is difficult to obtain a sufficient condition for a Hamiltonian cycle in a graph in terms of Hamiltonian properties of vertex-deleted subgraphs. While the problem of the existence of an undirected hypohamiltonian graph of a given order $n$ is not easy (and, in fact, unsolved for $n=17$ ), we have the following theorem:

Theorem 1.6.4 [Fouquet and Jolivet (1978), Grötschel and Wakabayashi (1978), Thomassen (1978)]. For every $n \geq 6$, there is a hypohamiltonian digraph of order $n$.

The hypohamiltonian digraphs in Thomassen (1978) have the additional interesting property of being planar. Also, hypohamiltonian oriented graphs were described in Thomassen (1978). He showed that the cartesian product $\mathbf{C}_{k} \times \mathbf{C}_{m-1}$ is hypohamiltonian. More generally, he proved that $\mathbf{C}_{k} \times \mathbf{C}_{m}$ is non-Hamiltonian whenever $k$ and $m$ are relatively prime. Subsequently, Erdös and Trotter (1978) determined exactly when $\mathrm{C}_{k} \times \mathbf{C}_{m}$ is Hamiltonian.

Theorem 1.6.5 [Erdös and Trotter (1978)]. Let $d=$ g.c.d. $(k, m)$. The cartesian product $\mathbf{C}_{k} \times \mathbf{C}_{m}$ of two cycles is Hamiltonian if and only if $d \geq 2$ and there exist positive integers $d_{1}, d_{2}$ such that $d_{1}+d_{2}=d$ and g.c.d. $\left(k, d_{1}\right)=$ g.c.d. $\left(m, d_{2}\right)=1$.

The cartesian product of cycles provides an infinite class of vertextransitive non-Hamiltonian digraphs. Only four such graphs are known in the 
undirected case, and Thomassen [see Bermond (1979)] has conjectured that only a finite number of such graphs exist. Babai (J.A. Bondy, private communication) disagrees. Lovász (1970) conjectured that every undirected vertex-transitive graph has a Hamiltonian path. This may be true for digraphs as well.

Grötschel, Thomassen, Wakabayashi (1980) showed that there is a hypotraceable digraph of order $n$ if and only if $n \geq 7$, and that, for each $k \geq 1$, there are infinitely many hypotraceable oriented graphs with precisely $k$ components.

A digraph is called homogeneously traceable if there is a Hamiltonian path starting at each vertex. Clearly, every hypohamiltonian digraph is homogeneously traceable. Thus, for every $n \geq 6$, there exist non-Hamiltonian homogeneously traceable digraphs. Bermond, Simoes-Pereira, and Zamfirescu (1979) have shown that non-Hamiltonian homogeneously traceable digraphs or order $n$ have at least $2 n$ arcs and exist if and only if $n \geq 5$. They also showed that non-Hamiltonian homogeneously traceable oriented graphs exist if and only if $n \geq 7$ and exhibited such digraphs with $2 n$ arcs.

Skupien and Wojda (1974) introduced the notion of strongly $(p, q)$ Hamiltonian graphs. This notion can be generalized to digraphs. A digraph $D=(X, U)$ is said to be strongly $q$-arc Hamiltonian if, for every system $\mathbb{S}$ of pairwise disjoint paths of the complete symmetric digraph with vertex set $X$ of total length $q$, the digraph $\mathbb{D}^{\prime}=(X, U \cup S)$ has a Hamiltonian cycle containing $S$. A strongly 1 -arc Hamiltonian digraph is strongly Flamiltonian-connected, that is, for every pair of vertices $x$ and $y$ there exists a Hamiltonian path from $x$ to $y$ and a Hamiltonian path from $y$ to $x$. Finally, we say that a digraph is strongly $(p, q)$-Hamiltonian if the digraph obtained by deleting any $r$ vertices is strongly $q$-arc Hamiltonian for all $r, 0 \leq r \leq p$. The following theorem generalizes Woodall's theorem (1.1.3).

Theorem 1.6.6 [Bermond (1975)]. If a digraph $D$ of order $n$ has the property that, for any two vertices $x$ and $y$, either $x$ dominates $y$ or $d^{+}(x)+d^{-}(y) \geq$ $n+p+q$, then $\mathbb{D}$ is strongly $(p, q)$-Hamiltonian.

As shown by Ghouila-Houri (1964, p. 324), the analogous generalization of his theorem (1.1.2) and, thus, of Meyniel's theorem (1.1.4) is not true. Indeed, there exist 2-connected digraphs in which each degree is at least $n+1$ and which contain arcs that belong to no Hamiltonian cycle. Other examples were also exhibited by Thomassen (1980a), where the following conjectures are given:

Conjecture 1.6.7 [Thomassen (1980a)]. Every 3-connected digraph of order $n$ and with minimum degree at least $n+1$ is strongly Hamiltonianconnected.

Conjecture 1.6.8 [Thomassen (1980a)]. Let $D$ be a 4-connected digraph of order $n$ such that the sum of the degrees of any pair of nonadjacent vertices is at least $2 n+1$. Then $D$ is strongly Hamiltonian-connected. 
3.1).

Thomassen (1980a) has proved this conjecture for tournaments (see Sec.

A digraph is weakly Hamiltonian-connected if, for any pair of vertices $x$ and $y$, there exists a Hamiltonian path from $x$ to $y$ or one from $y$ to $x$. Ghouila-Houri (1964) proved the following result which was rediscovered by Overbeck-Larisch (1976) in a slightly more general form.

Theorem 1.6.9 [Ghouila-Houri (1964)]. A 2-connected digraph of order $n$ and minimum degree at least $n+1$ is weakly Hamiltonian-connected.

Ghouila-Houri (1964) proved that the connectivity condition cannot be weakened in this theorem. Furthermore, Meyniel's theorem (1.1.4) cannot immediately be generalized, as there exist 2-connected tournaments that are not weakly Hamiltonian-connected. A complete characterization of weakly Hamiltonian-connected tournaments was given by Thomassen (1980a) (see Sec. 3.1).

\section{CYCLES OF PRESCRIBED LENGTHS}

\subsection{Pancyolic Digraphs}

Bondy (1972a) observed that conditions implying an undirected graph to be Hamiltonian often imply the graph to be pancyclic or to have a very special structure. Specifically, he proved that if a graph $G$ satisfies Ore's condition, then $G$ is pancyclic or isomorphic to $K_{n / 2, n / 2}$. The strongest result of this kind for undirected graphs is a result of Hakimi and Schmeichel (1974). The abovementioned result of Bondy was generalized to digraphs by Thomassen (1977).

Theorem 2.1.1 [Thomassen (1977)]. Let $D$ be a strong digraph of order $n$ such that, for any two nonadjacent vertices $x$ and $y$ of $D d(x)+d(y) \geq 2 n$. Then $D$ is pancyclic or $D$ is a tournament (in which case $D$ contains cycles of all lengths except 2) or else $n$ is even and $D$ is isomorphic to $\mathbb{K}_{n / 2, n / 2}^{*}$.

This theorem extends the aforementioned theorems of Camion, GhouilaHouri, and Woodall [ the fact that Ghouila-Houri's condition implies a digraph to be pancyclic or isomorphic to $K_{n / 2, n / 2}^{*}$ was first established by Häggkvist and Thomassen (1976)] and a result of Overbeck-Larisch (1977). However, it does not include Myniel's theorem, and it becomes false if we replace the degree condition by Meyniel's condition. To see this consider the digraph $D_{n . k}$ with vertex set $\left\{x_{1}, x_{2}, \ldots, x_{n}\right\}$ and arc set $\left\{\left(x_{i}, x_{j}\right) \mid i<j\right.$ or $\left.i=j+1\right\} \backslash$ $\left\{\left(x_{i}, x_{i+k-1}\right) \mid 1 \leq i \leq n-k+1\right\}$.Then $D_{n, k}$ satisfies Meyniel's condition and, moreover, it has only two pairs of nonadjacent vertices, $\left\{x_{1}, x_{k}\right\}$ and $\left\{x_{n-k+1}, s_{n}\right\}$, such that the inequality in Meyniel's theorem is an equality. For later purposes, we observe that the number of arcs of $D_{n, k}$ is $n(n-1) / 2+$ $k-2$. 
Bondy (1971) proved that every Hamiltonian undirected graph of order $n$ with at least $\frac{1}{4} n^{2}$ edges is pancyclic unless $n$ is even and the graph is isomorphic to $K_{n / 2, n / 2}$. He conjectured that the same is true for digraphs (when we replace $\frac{1}{4} n^{2}$ by $\frac{1}{2} n^{2}$ and $K_{n / 2, n / 2}$ by $K_{n / 2, n / 2}^{*}$ ). However, $D_{n, n-1}$ is Hamiltonian, but not pancyclic, and it has $\frac{1}{2} n(n+1)-3$ edges. On the other hand we have:

Theorem 2.1.2 [Häggkvist and Thomassen (1976)]. A Hamiltonian digraph with $n$ vertices and $\frac{1}{2} n(n+1)-1$ or more arcs is pancyclic.

Combining this theorem with 1.3.1 and 1.3.2, respectively, we get

Corollary 2.01.3 A strong digraph with $n$ vertices and $(n-1)(n-2)+3$ or more arcs is pancyclic.

Corollary 2.1 .4. A digraph with $n$ vertices and $(n-1)^{2}+1$ or more arcs is pancyclic.

The digraph $\mathbb{D}_{n, k}$ (defined above) with $k>n$, that is, the digraph with vertex set $\left\{x_{1}, x_{2}, \ldots, x_{n}\right\}$ and arc-set $\left\{\left(x_{i}, x_{j}\right) \mid i<j\right.$ or $\left.i=j+1\right\}$, has $\frac{1}{2} n(n+1)-1$ arcs and contains exactly one Hamiltonian cycle. Thus, the following theorem is best possible:

Theorem 2.1.5 [Müller and Pelant (1978)]. If a Hamiltonian digraph has $n$ vertices and $\frac{1}{2} n(n+1)$ or more arcs, then it has at least two distinct Hamiltonian cycles.

Müller and Pelant also gave conditions for the existence of $k$ distinct Hamiltonian cycles, when $3 \leq k \leq 6$. The analogous problem for undirected graphs was considered by Sheehan (1975).

Pancyclic tournaments are treated in Sec. 3.1.

2.2. The Minimum Number of Arcs Guaranteeing a Cycle of Length $k$ or at Least $k$

We first recall the state of the problem in the undirected case. The number of edges needed to ensure a cycle of length at least $k$ has been completely determined.

Theorem 2.2.1. [Woodall (1976)]. Given natural numbers $n$ and $k$, where $k \geq 3$, set $n=q(k-2)+r+1$, where $0 \leq r<k-2$. If $G$ is a graph of order $n$ with more than $\frac{1}{2} q(k-1)(k-2)+\frac{1}{2} r(r+1)$ edges, then $G$ contains a cycle of length at least $k$. 
Theorem 2.2.1 is best possible in view of the graph consisting of $q$ copies of $K_{k-1}$ and one copy of $K_{r+1}$, all having exactly one vertex in common. The case $n \equiv 1(\bmod k-2)$ was first solved by Erdös and Gallai (1959).

It is not completely known how many edges are needed in an undirected graph to ensure the existence of a cycle of length precisely $k$, when $k$ is even, not even for $k=4$ [for partial results see Bondy and Simonovits (1974)]. For $k$ odd, however, the problem is solved. Bondy (1971a) proved [by refining a result of Erdös (1963)] the following:

Theorem 2.2.2 [Bondy (1971a)]. If $n \geq 2 k-2$, then every graph of order $n$ with more than $n^{2} / 4$ edges contains a cycle of length $k$.

Woodall (1972) cleared up the situation for $k \leq n \leq 2 k-2$.

We now consider the analogous problem for digraphs.

Consider the following digraph $D$ of order $n$, where $n=q(k-1)+r$ and $0 \leq r<k-1: \mathbb{D}$ consists of the union of $q+1$ complete symmetric digraphs $H_{1}, H_{2}, \ldots, H_{q+1}$ such that $q$ of them have order $(k-1)$ and the last has order $r$. Then add all arcs of the type $(x, y)$, where $x \in V\left(\mathbb{H}_{i}\right), \mathrm{y} \in$ $V\left(H_{j}\right)$ with $i<j$. The resulting digraph $R_{n}$ has

$$
g(n, k)=\frac{1}{2} n(n-1)+\frac{1}{2}(n-r)(k-2)+\frac{1}{2} r(r-1)
$$

arcs, and contains no cycle of length $k$ or more.

Theorem 2.2.3 [Ḧ̈̈ggkvist and Thomassen (1976)]. A digraph of order $n$ with at least $g(n, k)$ arcs contains a cycle of length $k$ unless it is isomorphic to $R_{n}$.

This theorem was proved by Häggkvist and Thomassen (1976) only when $k-1$ divides $n$ but, as pointed out by Thomassen (1976), the proof can be modified to yield the theorem stated.

Lewin (1975) proved the weaker result that the assumption of Theorem 2.2.3 imples the existence of a cycle of length at least $k$.

The above problem is not completely solved when the digraph under consideration is strong. However, the following results are close to best possible.

Theorem 2.2.4 [Häggkvist and Thomassen (1976)]. Let $k$ be an integer, $k \geq$ 2. Then every strong digraph of order $n>(k-1)^{2}$ with more than $\frac{1}{2} n^{2}$ arcs contains a cycle of length $k$.

For $k$ odd, Theorem 2.2.4 is best possible except that the condition $n>(k-1)^{2}$ may be relaxed.

Theorem 2.2.5 [Häggkvist and Thomassen (1976)]. Let $k$ be an even 
integer, $k \geq 2$. Then every strong digraph of order $n$ with more than $\frac{1}{2} n(n-1)+\frac{1}{2}(k-1)(k-2)$ arcs contains a cycle of length $k$.

The digraph $D_{n, k}$ described after Theorem 2.1 .1 has $\frac{1}{2} n(n-1)+k-2$ arcs and contains no cycle of length $k$. Thus Theorem 2.2.5 is almost best possible.

The digraph $D_{n, k}$ has a strong oriented subgraph with $\frac{1}{2} n(n-3)+k-1$ arcs. This shows that the following result is best possible.

Theorem 2.2.6 [Heydemann (1980a)]. Let $k$ be an integer $k \geq 3$. Then every strong oriented graph of order $n$ with more than $\frac{1}{2} n(n-3)+k-1$ arcs contains a cycle of length $k$.

Let $D$ be the following strong digraph on $n$ vertices, where $n=q(k-2)+r+1,0 \leq r<k-2$. The vertex set of $D$ consists of the disjoint union of $k-2$ sets $X_{i}(i=1, \ldots, k-2), r$ of which are of cardinality $q+1$ and $k-2-r$ of cardinality $q$, plus one extra vertex $z$. The arcs of $D$ are all arcs of the form $(x, y)$ with $x \in X_{i}, y \in X_{j}, i<j$, and all the possible arcs incident with $z$. Then $\mathbb{D}$ is strong and contains no cycle of length at least $k$. The number $\phi(n, k)$ of arcs of $D$ is given by

$\dot{\phi}(n, k)=\frac{1}{2} n(n-1)+(n-\mathbb{1})-\frac{1}{2}(k-2-r) q(q-1)-\frac{1}{2} r q(q+1)$

or, equivalently,

$$
\begin{aligned}
\phi(n, k)= & \left(n^{2}(k-3)+2 n(k-1)\right. \\
& \left.-(k-2)(r+3)+r^{2}-1\right) / 2(k-2) .
\end{aligned}
$$

Let $D^{\prime}$ be the following strong digraph. The vertex set $X$ consists of two disjoint sets $A$ and $B$ together with an extra vertex $z$, with $|A|=k-3$, $|B|=n-k+2$. The arcs of $\mathbb{D}^{\prime}$ are all the arcs incident with $z$ together with all the arcs $(x, y)$ where either $x$ and $y$ belong together to $A$ or else $x \in B$ and $y \in A$. Then $\mathbb{D}^{\prime}$ is strong and contains no cycle of length at least $k$. The number $\psi(n, k)$ of arcs of $\mathbb{D}^{\prime}$ is given by

$$
\psi(n, k)=(k-1) n-2 k+4 \text {. }
$$

These two digraphs have been introduced by Bermond, Germa, Heydemann, and Sotteau (1980), where the following conjectures are given:

Conjecture 2.2.7 [Bermond, Germa, Heydemann, and Sotteau (1980)]. Let $D$ be a strong digraph of order $n$.

(i) If $n \geq 2 k-4$ and if $D$ has more than $\phi(n, k)$ arcs, then $D$ contains $\mathbb{2}$ cycle of length at least $k$.

(ii) If $k \leq n \leq 2 k-4$ and if $\mathbb{D}$ has more than $\psi(n, k)$ arcs, then $\mathbb{D}$ contains a cycle of length at least $k$. 
Note that, in view of the digraphs $D$ and $D^{\prime}$ described above, these conjectures, if true, are best possible. Conjecture 2.2.7 (ii) is true for $k=n$ by Theorem 1.3.1 and for $k=n-1$ by Bermond, Germa, Heydemann, and Sotteau (1980). Heydemann (private communication) has shown that it suffices to prove Conjecture 2.2.7 for $n=2 k-4$ and that the conjecture is true for $k \leq 5$. Also note that the examples above disprove Conjecture 6 in Thomassen (1979).

For oriented graphs the following holds:

Theorem 2.2.8 [Heydemann (1980)]. Given natural numbers $n$ and $k, n \geq$ $k \geq 3$, set $n=q(k-2)+r+1$, where $0 \leq r \leq k-3$. If $G$ is a strong oriented graph of order $n$ with more than $\frac{1}{2} n(n-1)-\frac{1}{2}(k-2-r) q$ $(q-1)-\frac{1}{2} r q(q+1)$ arcs, then $G$ has a cycle of length $k$ or more.

Theorem 2.2 .8 is best possible as demonstrated by any strong spanning oriented subgraph of the digraph showing that Conjecture 2.2.7(i) is best possible.

2.3. Conditions on the Degrees or the Chromatic Number Implying the Existence of a Cycle of Length $k$ or of Length at Least $k$.

Dirac (1952) proved that every 2-connected undirected graph with minimum degree at least $k$ contains a cycle of length at least $2 k$ or a Hamiltonian cycle. Generalizations have been given by Pósa (1963), Bondy (1971a) and Bermond (1976).

Ghouila-Houri (1964) showed that there exist 2-connected digraphs with arbitrarily high degrees and without cycles of length greater than 6 . For example, orient the complete bipartite graph $K_{|n-2 / 2| \cdot|n-1 / 2|}$ so as to produce no path of length 2 . The add two vertices which dominate and are dominated by all other vertices.

This example and various results on paths in digraphs prompted Bermond, Germa, Heydemann, and Sotteau (1980a) to conjecture the following:

Theorem 2.3.1 [Heydemann (1980)]. Let $D$ be a strong digraph of order $n$ such that, for any pair of nonadjacent vertices $x$ and $y$, we have $d(x)+d(y) \geq 2 n-2 h+1$. Then $D$ contains a cycle of length greater than or equal to $[(n-1) / h\rceil+1$.

Heydemann (1980) also obtained an analogous result for oriented graphs.

Bermond (1975) and Thomassen (1976) independently conjectured that if a. 2-connected digraph has order at least $2 k$ and minimum indegree and outclegree at least $k$, then it contains a cycle of length at least $2 k$. Recently, Thomassen (1980) showed that this conjecture is false for $n \geq 2 k+2$ (see counterexamples in Sec. 1.5 in connection with possible extensions of the Chvátal-Erdös theorem). However, the following holds: 
Theorein 2.3.2 [Thomassen (1981)]. A 2-connected digraph of order at least $k+2$ and with minimum indegree at least $k$, contains a cycle of length at least $k+2$.

The result is best possible in the sense that there exist infinitely many 2connected digraphs with minimum indegree and outdegree at least $k$ and whose longest cycles have length $k+2$. In the special case $n=2 k+1$, Thomassen (1981) verified the above conjecture using Theorem 1.4.2.

Theorem 2.3.3. [Thomassen (1981)]. If $\mathbb{D}$ is a 2-connected digraph of order $2 k+1$ such that every vertex has indegree and outdegree at least $k$, then $D$ contains a cycle of length at least $2 k$.

The following conjecture may hold:

Conjecture 2.3.4 [Thomassen (1979)]. If a digraph $D$ has minimum indegree and outdegree at least $k$ and if any two vertices of $D$ are on a common cycle, then $D$ contains either a cycle of length at least $2 k$ or a Hamiltonian cycle.

Perhaps stronger results can be obtained in the case of oriented graphs. Jackson (1980) conjectured the following:

Conjecture 2.3.5 [Jackson (1980)]. If $\mathbb{D}$ is a strong oriented graph with minimum indegree and outdegree at least $k$, then $D$ contains a cycle of length at least $2 k+1$.

Bondy (1976) obtained the next result on large cycles. It was conjectured by Las Vergnas (1976).

Theorem 2.3.6 [Bondy (1976)]. Every strong digraph with chromatic number $k$ contains a cycle of length at least $k$.

Theorem 2.3.6 generalizes the theorem of Gallai (1968) and Roy (1967) which states that a digraph of chromatic number $k$ has a path of length at least $k-1$. It also generalizes Camion's theorem (1.1.1).

\subsection{Cycles of Length at Most ks}

It is not known how many edges are needed in an undirected graph to ensure a. cycle of length at most $k$ (unless $k=3$ ). For digraphs, however, the problem has been completely solved by Bermond, Germa, Heydemann, and Sotteau (1980b), in response to a question of Thomassen. 
Theorem 2.4.1 [Bermond, Germa, Heydemann, and Sotteau (1980b)]. If $D$ is a strong digraph of order $n$ with at least $\frac{1}{2}\left(n^{2}+(3-2 k) n+k^{2}-k\right)$ arcs, then $D$ contains a cycle of length at most $k$.

This theorem is best possible. Indeed, let $D$ be the digraph obtained from a transitive tournament on $n-k+2$ vertices by replacing the arc joining the vertex of outdegree 0 in the transitive tournament to the vertex of outdegree $n-k+1$, by a path of length $k-1$ (using $k-2$ new vertices). Then $D$ is a strong digraph of order $n$ with $\frac{1}{2}\left(n^{2}+(3-2 k) n+k^{2}-k-2\right)$ arcs, and its smallest cycle has length $k+1$.

For diregular digraphs the following conjecture has been made.

Conjecture 2.4.2 [Behzad, Chartrand, and Wall (1970)]. If $D$ is an $r$ diregular strong digraph of order at most $k r$, then $D$ contains a cycle of length at most $k$.

Conjecture 2.4.2 is best possible in view of the digraph with vertex set $\left\{x_{0}, x_{1}, \ldots, x_{k r}\right\}$ and arc set $\left\{x_{i} x_{i+j} \mid 1 \leq j \leq r, 1 \leq i \leq k r\right\}$, where the indices are expressed modulo $k r+1$. The conjecture has been verified for $k=2,3$ by Behzad, Chartrand, and Wall (1970), for $r=2$ by Behzad (1973), for $r=3$ and some other values of $(r, k)$ by Bermond (1975a), for $r=4$ and for vertex-transitive digraphs by Hamidoune (1980a, 1980).

Caccetta and Häggkvist (1978) made the stronger conjecture that Conjecture 2.4.2 remains true if the diregularity condition is replaced by the weaker condition that every vertex has outdegree $r$ or more, and they verified this in the case $r=2$.

An extremal problem of a different nature involving small cycles in a digraph was considered by Chvátal and Thomassen (1978) (see the last section).

\section{CYCLES IN TOURNAMENTS}

\subsection{Hamiltonian Cycies}

Camion's theorem (1.1.1) was generalized by Harary and Moser (1966) who proved that a strong tournament contains cycles of all possible lengths. A slightly stronger result was obtained by Moon.

Theorem 3.1.1 [Moon (1968 p. 6)]. If $v$ is a vertex of a strong tournament of order $n$, and $k$ is any integer, $3 \leq k \leq n$, then the tournament has a cycle of length $k$ containing $v$.

Goldberg and Moon (1972) extended this by showing that each vertex of 
an $m$-arc-connected tournament is contained in at least $m$ cycles of each length.

For diregular tournaments there is an arc-version of Theorem 3.1.1:

Theroem 3.1.2 [Alspach (1967)]. If $e$ is an arc of a diregular tournament of order $n$, and $k$ is any integer, $3 \leq k \leq n$, then the tournament has a cycle of length $k$ containing $e$.

If tournament $T$ is obtained from a diregular tournament $T$ ' by reversing an arc of $T^{\prime}$, then the new arc need not be in a 3-cycle. However, in most cases the new arc must be in cycles of all possible lengths.

Theorem 3.1.3 [Alspach, Reid, and Roselle (1974)]. If tournament $T$ is obtained from a diregular tournament $\mathbb{T}^{\prime \prime}$ of order $n, n \geq 7$, by reversing an arc of $\mathbb{T}^{\prime}$, then the new arc is contained in cycles of $\mathbb{T}$ of all lengths $4,5, \ldots, n$.

Let the irregularity of a tournament be defined as $\max \mid d^{+}(x)$ $-d^{-}(x) \mid$ over all vertices $x$ of the tournament. If the irregularity is $1, T$ is said to be almost regular.

An analogue of Theorem 3.1.2 for almost regular tournaments is the following:

Theorem 3.1.4 [Jakobsen (1972)]. If $e$ is an arc of an almost regular toumament of order $n, n \geq 8$, and $k$ is any integer, $4 \leq k \leq n$, then $e$ is contained in a cycle of length $k$.

Theorems 3.1.2, 3.1.3, and 3.1.4 follow from the more general result below (with a little additional reasoning).

Theorem 3.1.5 [Thomassen (1980a)]. Let $\mathbb{T}$ be a toumament of order $n$ and irregularity $m$. Let $k$ be any integer, $4 \leq k \leq n$. If $n \geq 5 m+9$, then, for any two vertices $x$ and $y$, there is a path with $k$ vertices from $x$ to $y$. If $n \geq$ $5 m+3$, then any arc of $T$ is contained in a cycle of length $k$.

The proof of Theorem 3.1.5 depends on the fact that if $x, y, z$ are three vertices of a strong tournament with $n$ vertices and $k$ is any positive integer, $k \leq n-1$, the the tournament has a path of length $k$ connecting some two of $x, y, z$. That fact can be derived from the following theorem, which describes when two prescribed vertices are connected by paths of all possible lengths greater than 2 .

Theorem 3.1.6 [Thomassen (1980a)]. Let $x$ and $y$ be distinct vertices of a tournament $T$. Then $T$ has a Hamiltonian path connecting $x$ and $y$ unless one of (i), (ii), (iii), or (iv) below holds, in which case $T$ has no such Hamiltonian path.

(i) $\mathbb{T}$ is not strong, and the initial or terminal component contains neither $x$ nor $y$. 
(ii) $T$ is strong, $T-x$ is not strong, and $y$ belongs to an intermediate component of $T-x$.

(iii) $T$ is strong, $T-y$ is not strong, and $x$ belongs to an intermediate component of $T-y$.

(iv) $T$ is isomorphic to $T_{6}^{s}$ or $\bar{T}_{6}^{s}$ of Figure 4.

Furthermore, if none of (i), (ii), (iii), and (iv) holds, then, for every integer $k, 3 \leq k \leq n-1, T$ has a path of length $k$ connecting $x$ and $y$.

It is not known precisely, when a given arc is contained in a Hamiltonian cycle of a tournament. The following is a sufficient condition for this.

Theorem 3.1.7 [Thomassen (1980a)]. In a 3-connected tournament every arc is contained in a Hamiltonian cycle.

The tournaments $T_{6}^{s}$ and $\bar{T}_{6}^{s}$ of Figure 4 show that there are 2-connected tournaments with arcs that are not contained in any Hamiltonian cycle. Thomassen (1980a) described an infinite family of such tournaments. Furthermore, he showed that every 4-connected tournament is (strongly) Hamiltonian connected, and that there are infinitely many 3-connected ones that are not.

Rédei (1934) proved that the number of Hamiltonian paths in a tournament is odd. However, Camion (1973) showed that the parity of the number of Hamiltonian cycles can always be changed by reversing a well-chosen arc. For a problem on arc-reversal, see Conjecture 5.6.

Szele (1966) showed that if $h_{p}(n)$ is the maximum number of Hamiltonian paths in a tournament of order $n$, then

$$
\frac{1}{2} \leq \lim _{n \rightarrow \infty}\left[\frac{h_{p}(n)}{n !}\right]^{1 / n} \leq 2^{-3 / 4}
$$

Since $h_{\mathrm{p}}(n-2) \leq h_{c}(n) \leq h_{p}(n)$, where $h_{c}(n)$ is the maximum number of
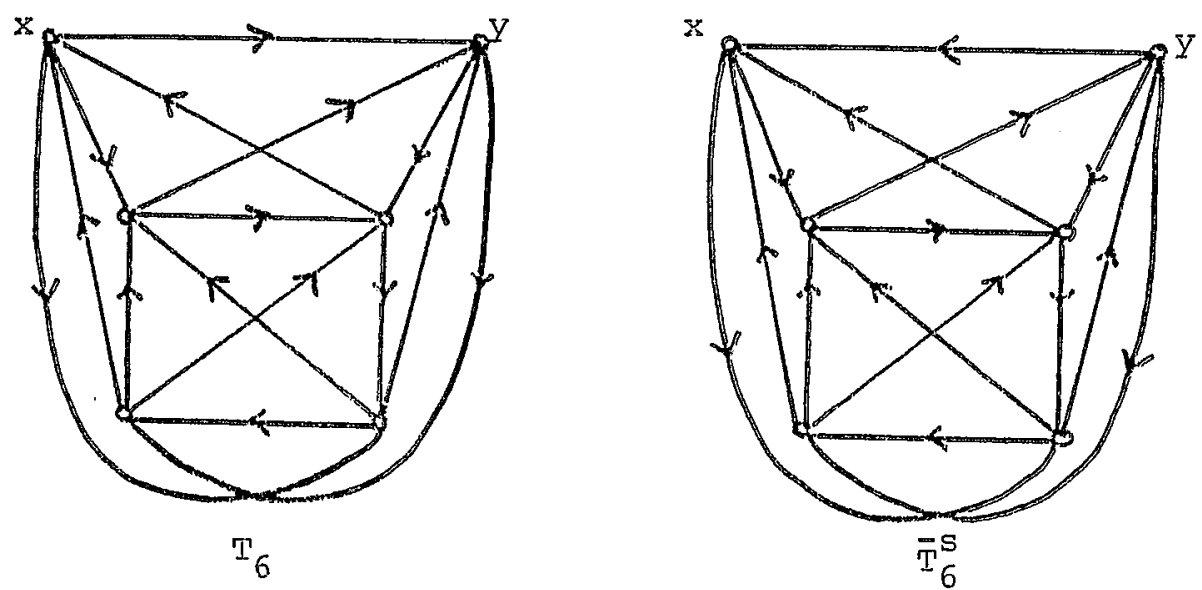

FIGURE 4. Toumaments with no Hamiltonian path connecting $x$ and $y$. 
distinct Hamiltonian cycles in a tournament with $n$ vertices, Szele's result remains true if we replace $h_{p}(n)$ by $h_{c}(n)$.

Grünbaum (1969a) conjectured that, for every odd integer $k, 1 \leq k \leq$ $h_{p}(n)$, there is a tournament with $n$ vertices and precisely $k$ distinct Hamiltonian paths. Thomassen (1980b) disproved this by observing that there is no tournament with precisely 7 distinct Hamiltonian paths. Perhaps the following holds:

Conjecture 3.1.8 [Grünbaum (1969a)]. For every integer $k, 1 \leq k \leq$ $h_{c}(n)$, there is a tournament with $n$ vertices and precisely $k$ distinct Hamiltonian cycles.

Thomassen (1980b) proved the weaker statement that, for every integer $k \geq 1$, there is a tournament with precisely $k \mathbb{H a m i l t o n i a n}$ cycles.

Moon (1966) proved that the minimum number of Hamiltonian paths in a strong tournament of order $n$ increases exponentially with $n$. From that fact the following was established:

Theorem 3.1.9 [Thomassen (1980b)]. There exists a constant $c>1$ such that every 2-connected tournament of order $n$ has at least $c^{\prime \prime}$ Hamiltonian cycles and such that every strong tournament of minimum outdegree at least $k$, where $k \geq 2$, contains at least $c^{k}$ Hamiltonian cycles.

The last part of Theorem 3.1.9 extends the aforementioned result of Goldberg and Moon (1972), that a $k$-arc connected tournament has at least $k$ Hamiltonian cycles.

The tournaments with exactly one Hamiltonian cycle were characterized and enumerated by Douglas (1970), Garey (1972), and Egorycev (1974).

\subsection{Cycles of Prescribed Lengths}

Let $C(n, k)$ denote the maximum number of distinct cycles of length $k$ in a tournament of order $n$.

Theorem 3.2.1 [Korvin (1967)].

$$
\left(\begin{array}{l}
n \\
k
\end{array}\right) \frac{(k-1) !}{2^{k}} \leq C(n, k) \leq(k+1)\left(\begin{array}{l}
n \\
k
\end{array}\right) \frac{(k-1) !}{2^{(3 / 4)^{k-3}}}
$$

For $k=3,4$, the exact values of $C(n, k)$ are given in the following result:

Theorem 3.2.2 [Kendall and Babington Smith (1940)]. The number of 3cycles in a tournament with outdegrees $s_{1}, s_{2}, \ldots, s_{n}$ equals 


$$
\left(\begin{array}{l}
n \\
3
\end{array}\right)-\sum_{i=1}^{n}\left(\begin{array}{l}
s_{i} \\
2
\end{array}\right)
$$

and

$$
C(n, 3)= \begin{cases}\frac{n\left(n^{2}-1\right)}{24}, & \text { if } n \text { is odd } \\ \frac{n\left(n^{2}-4\right)}{24}, & \text { if } n \text { is even. }\end{cases}
$$

Theorem 3.2.3 [Beineke and Harary (1965)].

$$
C(n, 4)=\frac{1}{2}(n-3) C(n, 3) \text {. }
$$

Furthermore, Berman (1975) found an upper bound for the number of 5 cycles in toumaments of a special type.

Reid proved that if $l<k$, then the existence of sufficiently many $l$-cycles in a tournament also implies a $k$-cycle.

Theorem 3.2.4 $\llbracket \mathbb{R}$ eid (1971)]. Let $3 \leq l<k \leq n$ and let $n=q(k-1)+r$, where $0 \leq r \leq k-2$. The maximum possible number of $l$-cycles in a tournament of order $n$ with no $k$-cycle is given by $q \mathbb{C}(k-\mathbb{1}, l)+\mathbb{C}(r, l)$.

Moon (1968, p. 10) showed that a strong tournament contains at least $n-k+1 k$-cycles for $3 \leq k \leq n$. Las Vergnas refined this result. We denote by $A_{n}$ the tournament with vertex set $\left\{x_{1}, x_{2}, \ldots, x_{n}\right\}$ and arc set $\left\{\left(x_{i}, x_{j}\right) \mid i<j-1\right.$ or $\left.i=j+1\right\}$.

Theorem 3.2.5 [Las Vergnas (1975)]. If $T$ is a strong tournament of order $n$ which is not isomorphic to $A_{n}$, then $T$ contains at least $n-k+2 k$-cycles for $4 \leq k \leq n-1$

As a corollary, a strong tournament of order $n$ which is not isomorphic to $A_{n}$ contains at least $\left(\begin{array}{l}n \\ 2\end{array}\right)-3$ cycles. Las Vergnas also extended Theorem 3.2 .5 to complete digraphs.

Las Vergnas has generalized some results on cycles in tournaments by considering digraphs of the form $D_{1} \cup D_{2} \cup W$, where $D_{1}$ and $D_{2}$ are disjoint digraphs and $W$ is a set of arcs between $\mathbb{D}_{1}$ and $\mathbb{D}_{2}$. Among other things, he proved the following theorem:

Theolem 3.2.6 [Las Vergnas (1973)]. Let $D$ be a digraph of order $n$ of the form $D_{1} \cup D_{2} \cup W$, where $D_{1}$ and $D_{2}$ are disjoint cycles and $W$ is a set of 
arcs between $D_{1}$ and $D_{2}$ such that any vertex of $D_{1}$ is adjacent to any vertex of $D_{2}$. If $D$ is strong and $k$ is any integer, $3 \leq k \leq n$, then $D$ contains a cycle of length $k$.

Beineke and Little (1980) and Jackson (1981) have investigated cycles in bipartite tournaments, i.e., complete bipartite oriented graphs.

Theorem 3.2.7 [Jackson (1981)]. A strong bipartite tournament has a cycle of length $2 k$ or more if, for any two adjacent vertices $x$ and $y$, either $x$ dominates $y$ or $d^{+}(x)+d^{-}(y) \geq k$.

Beineke and Little (1980) proved that, if a bipartite tournament has a cycle of length $2 k$, then it has cycles of all smaller lengths unless $k$ is even and the $2 k$-cycle induces a special digraph.

Long cycles in $k$-partite tournaments have been studied by Ayel (1980).

\subsection{Generalized Cycles}

If $e=\left(e_{1}, e_{2}, \ldots, e_{k}\right)$ is a $k$-tuple of 1 's and -1 's, then an $e$-cycle is a digraph whose underlying undirected graph is a cycle, $\left(v_{1}, v_{2}, \ldots, v_{k}, v_{1}\right)$, such that $v_{i}$ dominates $v_{i+1}$ if and only if $e_{i}=1$ (here $v_{k+1}=v_{1}$ ). If $k$ is even and $e_{i}=(-1)^{i}$, an $e$-cycle is called an antidirected cycle. An e-path and an antidirected path are defined analogously. Grünbaum (1971) proved that every tournament, with three exceptions (of orders 3,5, and 7, respectively), has an antidirected Hamiltonian path, and made the following conjecture:

Conjecture 3.3.1 [Grünbaum (1971)]. Let $n$ be an even integer, $n \geq 10$. Then every tournament of order $n$ contains an antidirected Hamiltonian cycle.

The conjecture was first proved by Thomassen (1973a) for $n \geq 50$. Then Rosenfeld (1974) obtained the following stronger result

Theorem 3.3.2 [Rosenfeld (1974)]. Every tournament of even order $n, n \geq$ 28, has an antidirected Hamiltonian cycle.

He also made the following conjecture:

Conjecture 3.3.3 [Rosenfeld (1974)]. There is an integer $n_{0}$ such that, for every tournament of order $n, n \geq n_{0}$, and every $n$-tuple $e$ of 1 's and -1 's, $e \neq(1,1, \ldots, 1)$ and $e \neq(-1,-1, \ldots,-1), T$ contains an $e$-cycle.

Forcade (1973) proved that the parity of the number of embeddings of an $\left(e_{1}, e_{2}, \ldots, e_{n}\right)$-cycle in a tournament of order $n$ depends only on $\left(e_{1}, e_{2}, \ldots\right.$, $\left.e_{n}\right)$ and the number of Hamiltonian cycles of the tournament. 


\section{PARTITIONS, PACKINGS, AND COVERINGS BY CYCLES}

In this section we are primarily concerned with packing and covering the arcs of a digraph by cycles. A packing is a set of arc-disjoint cycles of the digraph. A covering is a set of cycles covering all the arcs of a digraph. If a digraph has a packing which is also a covering, we say that the arcs of the digraph can be partitioned into cycles.

\subsection{Partitions}

The first general result is Veblen's theorem:

Theorem 4.1.1 The arcs of a digraph can be partitioned into cycles if and only if, for each vertex $x, d^{+}(x)=d^{-}(x)$.

Meyniel (private communication) has conjectured that there always exists such a decomposition into at most $(n-1)$ cycles, where $n$ is the order of the digraph.

It should be noted that Veblen's theorem is a consequence of Euler's theorem:

Theoren 4.1.2. A strong digraph admits a closed walk containing all the arcs if and only if for each vertex $x, d^{+}(x)=d^{-}(x)$.

For diregular digraphs, a more precise result has been obtained by Kotzig. A 2-factor in a digraph is a disjoint union of cycles covering all the vertices of $\mathbb{D}$.

Theorem 4.1.3 [Kotzig (1969)]. The arcs of a diregular digraph can be partitioned into 2-factors.

It is well known that the edges of the complete (undirected) graph $\mathbb{K}_{2 n+1}$ can be partitioned into Hamiltonian cycles. The analogous result for complete symmetric digraphs has been proved by Tillson (1980).

Theorem 4.1.4 [Tillson (1980)]. If $n \neq 4,6$, then the $\operatorname{arcs}$ of $\mathbb{K}_{n}^{*}$ can be partitioned into Hamiltonian cycles.

For $n=4,6$, such a decomposition is impossible. Theorem 4.1.4 was conjectured by Bermond and Faber (1976), who discussed relations between decompositions and sequenceable groups and other combinatorial objects. An analogous result for bipartite complete symmetric digraphs is as follows:

Theorem 4.1.5 [Bermond and Faber (1976)]. The arcs of $K_{n, n}^{*}$ can be partitioned into Hamiltonian cycles. 
Another possible generalization of the decomposition of $K_{2 n+1}$ into Hamiltonian cycles is described in the following conjecture due to P.J. Kelly [see Moon (1968, p. 7)].

Conjecture 4.1.6 [Kelly]. The arcs of a diregular tournament can be partitioned into Hamiltonian cycles.

Kelly's conjecture has been verified for tournaments of order 9 or less by Alspach (private communication). Thomassen (1980c) proved that, for some positive constant $c$, every diregular tournament of order $n$ has $c \sqrt{n}$ arcdisjoint Hamiltonian cycles (see Theorem 1.4.8). As a consequence of Kelly's conjecture, an oriented graph is Hamiltonian, if it is obtained from a diregular tournament of order $n$ by deleting any $\frac{1}{2}(n-3)$ arcs. Thomassen (1980c) proved the following related result.

Theorem 4.1 .7 [Thomassen (1980c)]. If $T$ is a $5 k$-connected tournament and $A$ is a set of at most $k$ arcs of $T$, then $T-A$ is Hamiltonian,

If $T$ is a diregular tournament of order $n$, then $T$ has connectivity at least $n / 3$, as observed by Thomassen (1980a), and hence, by Theorem 4.1.7, $T-A$ is Hamiltonian if $A$ is a set of at most $[n / 15]$ arcs.

Thomassen (1980c) made several other conjectures related to Kelly's conjecture, for example the following:

Conjecture 4.1.8 [Thomassen (1980c)]. There exists a function $f(k)$ such that every $f(k)$-connected tournament has $k$ arc-disjoint Hamiltonian cycles.

By Camion's theorem (1.1.1), $f(1)=1$, and Thomassen (1980c) gave examples showing that $f(2)>2$. He conjectured that $f(2)=3$.

As a $k$-diregular tournament has $2 k+1$ vertices, Bondy (1978) conjectured that every $k$-diregular digraph of order $2 k+1$, except $\mathbb{D}_{5}$ and $\mathbb{D}_{7}$ (see $\mathbb{F i g} .3$ in Sec. 1.4) can be decomposed into Hamiltonian cycles. Thomassen (1980) disproved this conjecture as follows. Let $k$ be either 3 or 5. Take two disjoint copies of $K_{k}^{*}$, add a new vertex which dominates all vertices of one of the copies of $K_{k}^{*}$, and which is dominated by all vertices of the other copy, the add $k$ arcs such that the resulting digraph is $k$-regular. This digraph cannot be decomposed into Hamiltonian cycles, because $\mathbb{K}_{3}^{*}$ and $K_{5}^{*}$ cannot be decomposed into Hamiltonian paths (by 4.1.4). No infinite family of counterexamples is known, so Bondy's conjecture may be true for $k$ sufficiently large.

Jackson (1980a) made the following conjecture which, if true, implies the truth of Kelly's conjecture:

Conjecture 4.1.9 [Jackson (1980a)]. Every oriented graph of order $n$ such that $d^{+}(x)=d^{-}(x)$ for each vertex $x$, can be decomposed into at most $\lfloor n / 2\rfloor$ arc-disjoint cycles. 
Finally, note that other possible attacks of Kelly's conjecture are given by Conjectures 1.4.5 and 1.4.6.

Jackson (1980) proposed a bipartite version of Kelly's conjecture which is also of interest in connection with Theorem 4.1.5.

Conjecture 4.1.10 [Jackson (1980)]. Every diregular complete bipartite oriented graph can be partitioned into Hamiltonian cycles.

It follows from Theorem 3.2.7 that any such oriented graph has a Hamiltonian cycle.

In the undirected case Hamiltonian decompositions have been obtained for products of special graphs [see Bermond (1978)]. Baranyai and Százs (1981) proved that if $G_{1}$ and $G_{2}$ are two undirected graphs decomposable into Hamiltonian cycles, then their lexicographic product is also decomposable. The analogous result for digraphs is perhaps also true.

\subsection{Packings and Coverings}

If the vertex set of a digraph $D$ is partitioned into nonempty sets $A$ and $B$, and no vertex of $B$ dominates any vertex of $A$, than the set of $\operatorname{arcs}$ from $A$ to $B$ forms a cocircuit of $D$. Lucchesi and Younger (1978) [see also Lovász (1976)] have proved that the maximum number of arc-disjoint cocircuits of a digraph is equal to the minimum number of arcs meeting all the cocircuits. From this, the following can be deduced:

Theorem 4.2.1 [Lucchesi and Younger (1978)]. For a planar digraph, the maximum number of arc-disjoint cycles equals the minimum number of arcs meeting all cycles.

This minimax relation does not hold in general, as can be seen by considering an appropriate orientation of $K_{3,3}$ (Fig. 5).

Kotzig (1975) conjectured that Theorem 4.2.1 does not even extend to tournaments and this was verified by Bermond and Kodratoff (1976) [see
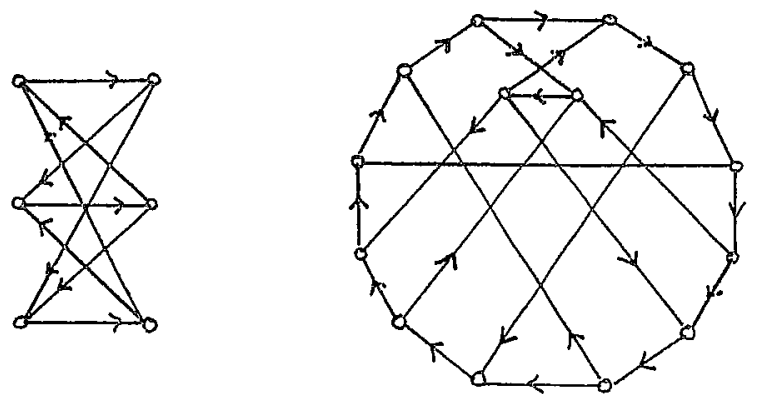

FIGURE 5. Two oriented graphs with no two arc-disjoint cycles such that every arcdeleted subgraph has a cycle. 
also Bermond (1975b)]. Kotzig's conjecture also follows from a result found by Erdös and Moon (1965) [and improved by Jung (1970)] concerning large acyclic subgraphs in tournaments. Let $v(D)$ be the maximum number of arcdisjoint cycles of a digraph $D$, and let $\tau(D)$ be the minimum number of arcs meeting all cycles of $D$ (that is, their deletion results in an acyclic digraph). Clearly, $\tau(D) \geq v(D)$. Now let $f(n)=\max \tau\left(T_{n}\right)$ and $\pi(n)=\max v\left(T_{n}\right)$, where the maximum is taken over all tournaments of order $n$. Thus, $\pi(n)$ is also equal to the maximum number of edge-disjoint cycles of the complete (undirected) graph. By a result of Chartrand, Geller, and Hedetniemi (1971),

$$
\pi(n)=\left\lfloor\frac{n}{3}\left\lfloor\frac{n-1}{2}\right\rfloor\right\rfloor
$$

The fact that Theorem 4.2.1 fails for tournaments is established by the next theorem.

Theorem 4.2.2 [Bermond (1975b), Bermond and Kodratoff (1976)]. For $n \geq 10, f(n)>\pi(n)$.

The proof of Theorem 4.2.2 depends on the determination of $\tau\left(D_{1} \otimes \mathbb{D}_{2}\right)$, where $D_{1} \otimes D_{2}$ is the lexicographic product of digraphs $D_{1}$ and $D_{2}$. The following result was conjectured by Bermond (1975b):

Theorem 4.2.3 [Thomassen (1975)].

$$
\tau\left(\mathbb{D} \otimes \mathbb{D}_{2}\right)=\left|V\left(D_{1}\right)\right| \tau\left(D_{2}\right)+\left|V\left(D_{2}\right)\right|^{2} \tau\left(D_{1}\right)
$$

The analogous statement for $v\left(D_{1} \otimes D_{2}\right)$ is not true in general. Bermond (1975b) conjectured $v\left(D_{1} \otimes S_{q}\right)=q^{2} v\left(D_{1}\right)$ where $S_{q}$ is a digraph on $q$ vertices without arcs. That has been disproved by Sterboul (private communication) with $q=2$ and with $D_{1}$ equal to the digraph of Figure 6 .

In the undirected case, the problem of determining the minimum number $\mathrm{f}(n, k)$ of edges such that every graph $G$ on $n$ vertices contains $k$ pairwise edge-disjoint cycles is an unsolved problem. The following exact values are known: $f(n, 2)=n+4$ [Erdös and Pósa (1962)]: $f(n, 3)=n+10$ [Moon (1964)]; $f(n, 4)=n+18$ [Häggkvist (1975)]. Using the Four-Colour Theorem, Häggkvist (1975) has solved the analogous problem for planar graphs completely. For digraphs, this problem is easily solved. Indeed, it is obvious that, for $k \geq 2$, every digraph with at least $n(n-1) / 2+k$ arcs contains $k$ pairwise arc-disjoint cycles and it is easy to see that this is best possible.

Finally, we mention that Nash-Williams (1969) has conjectured that a digraph with minimum indegree and outdegree at least $n / 2$ contains two arcdisjoint Hamiltonian cycles. 


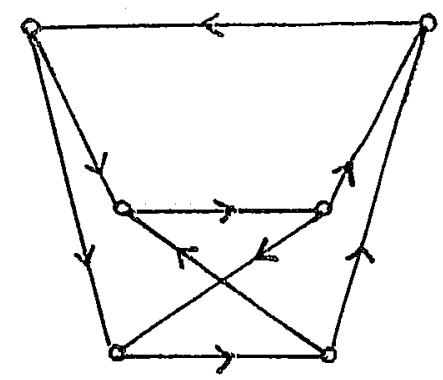

FIGURE 6. A counterexample to a conjecture of Bermond (1975b).

As pointed out by Thomassen (1980c), the results in Thomassen (1981) imply this conjecture for $n$ odd. However, Ninćak (1973) gave a counterexample for $n=6$ and the conjecture is open for each even $n \geq 8$.

\subsection{Partitions, Packings, and Coverings with Cycles of given Length}

The problem of decomposing a digraph into cycles of a given length is related to design theory, in particular when one is concerned with decompositions of $K_{n}^{*}$. The following conjecture has been proposed:

Conjecture 4.3.1 [Bermond (1975)]. The arcs of $K_{n}^{*}$ can be partitioned into cycles of length $k, k \leq n$, if and only if $n(n-1) \equiv 0(\bmod k)$, except when $n=k=4, n=k=6$, and $n=6, k=3$.

Note that for the three exceptional pairs of values no such partition exists. Many particular cases of the conjecture have been solved, one of them being Tillson's theorem (4.1.4) for $k=n$. For a comprehensive survey of these results, the reader is referred to Sotteau (1980a). For further results and more details, see also Bermond and Sotteau $(1976,1978)$ and Bermond, Huang, and Sotteau (1978).

For complete symmetric bipartite digraphs the situation is clarified by the following result, conjectured by Bermond and Faber (1976):

Theorem 4.3.2 [Sotteau (1980)]. The arcs of $K_{n, m}^{*}$ can be partitioned into cycles of length $2 k$ if and only if $n \geq k, m \geq k$, and $n m \equiv 0(\bmod k)$.

Analogous problems for packings or coverings of $K_{n}^{*}$ with cycles of a given length $k$ can also be considered. Partial results have been obtained, in particular, for $k=3$ [Bermond (1975)].

\section{FURTHER PROBLEMS INVOLVING CYCLES}

Not much is known about antidirected cycles in digraphs (for definitions see 3.3). Häggkvist (1977a) conjectured that every digraph of order $n$ with more 
than $3(n-1)$ arcs contains an antidirected cycle. Recently, this conjecture was disproved independently by L.D. Andersen (private communication) and by Lehel (1980). The exact bound is still to be determined; however, the following partial result has been obtained.

Theorem 5.1 [Grant, Jaeger, and Payan (1979)]. Any digraph of order $n$ with more than $7(n-1) / 2$ arcs has an antidirected cycle.

Grant, Jaeger, and Payan (1979) proved, also, that every digraph without antidirected cycles has a vertex of degree less than or equal to 5 , and conjectured that such graphs are 5-colorable.

Marcus (1979) has considered the problem of describing those constants $a_{k}, b_{k}$ for which the following holds: every $k$-connected digraph of order $n$ with $m$ or more arcs contains a strong spanning subgraph with at most $a_{k} m+b_{k}(n-1)$ arcs. He has shown that the truth of the following conjecture would yield a complete solution for the case $k=2$ :

Conjecture 5.2 [Marcus (1979)]. Any 2-connected digraph contains a cycle with two chords (i.e., two arcs joining two vertices of the cycle).

Corrádi and Hajnal (1963) proved that every undirected graph of order at least $3 k$ and minimum degree at least $2 k$ contains $k$ pairwise disjoint cycles. We propose an analogous conjecture for digraphs.

Conjecture 5.3. A digraph with minimum outdegree at least $2 k-1$ contains $k$ pairwise disjoint cycles.

The complete digraph of order $2 k-1$ shows that the bound is necessary. The conjecture is trivial for $k=1$, and it has been verified by Thomassen for $k=2$.

The following conjecture on arc-disjoint cycles, due to D.H. Younger [private communication (1973)] is of interest in connection with Theorem 4.2.1.

Conjecture 5.4 [Younger (private communication)]. There exists a function $f(k)$ such that any digraph contains either $k$ arc-disjoint cycles or a set of $f(k)$ arcs meeting all cycles.

Younger (1973) described the graph of order 14 in Figure 5 showing that $f(2)>2$. He conjectured that $f(2)=3$.

The analogous statement for undirected graphs was proved by Erdös and Pósa (1962).

Using standard operations on digraphs, it is easy to reformulate Conjecture 5.4 as follows: 
Conjecture 5.5 There exists a function $g(k)$ such that any digraph contains either $k$ disjoint cycles or a set of $g(k)$ vertices meeting all cycles.

Again, the analogous result for undirected graphs was proved by Erdös and Pósa (1965). The question of the existence of $g(2)$ was originally posed by Gallai (1968a).

Younger (1973) and Kosaraju (1977) conjectured that $g(2)=3$. Kosaraju (1977) proved that, if any three cycles of a digraph have a vertex in common, then all cycles have a common vertex.

The problem of finding many disjoint cycles covering all vertices in a special class of digraphs, called de Bruijn Graphs, has been discussed by Lempel (1971).

Gyori (1978) described the digraphs with the property that any arc belongs to at most two cycles. He also described those with the property that any vertex is contained in at most three cycles and extended, thereby, an earlier result of Adám (1976).

It is difficult to count the number of cycles in a digraph unless one is interested only in special digraphs (for example 3-cycles in a tournament). In this connection, the following conjecture is of interest:

Conjecture 5.6 [Adám (1964)]. If a digraph $D$ contains at least one cycle, then it is possible to reverse the direction of an arc so as to obtain a digraph with fewer cycles than $D$.

Chvátal and Thomassen proved the following result on local girth of orientation.

Theorem 5.7 [Chvátal and Thomassen (1978)]. There exists a function $h(k)$ such that any undirected graph $G$ has an orientation $D$ with the property that any edge which in $G$ is contained in a cycle of length at most $k$, is contained in $D$ in a cycle of length at most $h(k)$.

In Theorem 5.7, the object is to obtain many small cycles. On the other hand, one might try to orient the edges of an undirected graph so as to create no small cycles. This is nontrivial only if we insist that the resulting digraph be strong. We propose the problem of characterizing the 2-edge-connected graphs $G$, such that, for any strong orientation $D$ of $G$, the (directed) girth of $D$ equals the girth of $G$. The following graphs enjoy this property: any graph of girth $g$ such that every cycle of length greater than $g$ has a chord (for example, a complete bipartite graph), any graph with $n$ vertices and maximum degree $n-1$, any planar triangulation, and, finally, the Petersen graph [Chvátal and Thomassen (1978)].

It is easy to prove that a strong digraph has an odd cycle unless it is bipartite [see, for example, Harary, Norman; and Cartwright (1965)]. Also, 
It is easy to show that an undirected 2 -connecrea grapn nais an even cyule unless it is an odd cycle. However, it seems difficult to decide whether or not a digraph has an even cycle. Younger (private communication) has proposed the problem of describing an efficient algorithm for finding such a cycle, and Lovász has made the following conjecture:

Conjecture 5.8 [Lovász, see Koh (1976)]. There exists a natural number $k$ such that every digraph with minimum indegree and outdegree at least $k$ has an even cycle.

Koh (1976) has shown that $k$ must be at least three by exhibiting an infinite familty of counterexamples with $k=2$.

A well-known theorem of Whitney (1932) asserts that two graphs $G$ and $H$ are isomorphic if $G$ is 3-connected and there exists a cycle-preserving bijection of the edge set of $G$ onto the edge set of $H$. This result is not true for digraphs in general. However, for tournaments, the following holds:

Theorem 5.9 [Goldberg and Moon (1971)]. Let $\mathbb{T}$ and $\mathbb{T}^{\prime}$ be strong toumaments with arc sets $U$ and $U^{\prime}$, respectively. If there exists a bijection of $U$ onto $U^{\prime}$ which preserves 3-cycles and 4-cycles, then $\mathbb{T}$ is isomorphic to $\mathbb{T}^{\prime}$ or its converse.

Goldberg and Moon (1971) pointed out that Theorem 5.9 is not valid if the bijection only preserves 3-cycles, but that it may be true if the bijection only preserves 4-cycles. Other problems of this type have been considered by Waldrop (1978).

\section{ACKNOWLEDGEMENTS}

The authors wish to thank J.A. Bondy and K.B. Reid for their many valuable remarks on the paper. In addition, we appreciate greatly the comments by J.-L. Fouquet, M.-C. Heydemann, J.W. Moon, R.C. Read, D.H. Younger, and three anonymous referees.

\section{BIBLIOGRAPHY}

Adam, A., Problem 2 in: Theory of graphs and its applications, Proc. Coll. Smolenice, Czech. Acad. Sci. Publ. (1964), 157.

Adám, A., On graphs satisfying some conditions for cycles. Acra Cybernet. 3(1976), 3-13.

Alpern, S., Superhamiltonian graphs. J. Combinatorial Theory $B 25$ (1978), 62-73.

Alspach, B., Cycles of each length in regular tournanents. Canad. Math. Bull. 10(1967), 283-286. 
Alspach, B., K. B. Reid, and D. P. Roselle, Bypasses in asymmetric digraphs. $J$. Combinatorial Theory $B$ 17(1974), 11-18.

Ayel, J., Long paths and cycles in oriented p-partite graphs. Preprint (1980).

Baranyai, Z. and G. R. Szász, Hamiltonian decomposition of lexicographic product. J. Combinatorial Theory (1981). To appear.

Behzad, M., Minimal 2-regular digraphs with given girth. J. Math. Soc. Japan 25(1973), 1-6.

Behzad, M., G. Chartrand, and C. E. Wall, On minimal regular digraphs with given girth. Fund. Math. 69(1970), 227-231.

Beineke, L. W. and C. H. C. Little, Cycles in complete oriented bipartite graphs. Preprint (1980).

Beineke, L. W. and F. Harary, The maximum number of strongly connected subtournaments. Canad. Math. Bull. 8(1965), 491-498.

Beineke, L. W. and K. B. Reid, Tournaments, Selected Topics in Graph Theory. Edited by L. W. Beinecke and R. J. Wilson. Academic Press, New York (1979).

Bellmore, H. and G. Nemhauser, The Travelling Salesman Problem: A Survey, Oper. Res. 16(1968), 538-558.

Berman, D. M. On the number of 5-cycles in a tournament. Proc. 6th Southeastern Conf. on Combinatorics, Graph Theory and Computing. Boca Raion, Utilitas Math., Congressus Numerantium XIV (1975), $101-108$.

Bermond, J. C., Cycles dans les graphes et G-configurations. Thesis, University of Orsay (1975).

Bermond, J. C., 1-graphes réguliers minimaux de girth donné, Proc. Journées franco-belges sur les graphes et hypergraphes. Cahiers du CERO, Bruxelles, 17(1975a), 125-135.

Bermond, J. C., The circuit hypergraph of a tournament, Infinite and finite sets. Colloq. Math. Soc. János Bolyai 10. North Holland (1975b), $165-180$.

Bermond, J. C., On Hamiltonian walks. Proc. 5th British Combinatorial Conf., Aberdeen, Congressus Numerantium XV. Utilitas Math. Publ. (1976), 41-51.

Bermond, J. C., Hamiltonian decompositions of graphs, directed graphs, and hypergraphs. Advances in Graph Theory. Edited by B. Bollobás. Ann. Discrete Math. 3(1978), 21-28.

Bermond, J. C., Hamiltonian Graphs, Selected Topics in Graph Theory. Edited by L. W. Beineke and $\mathbb{R}$. J. Wilson. Academic, New York (1979), 127-167.

Bermond, J. C., Problem in: Proc. Coll. Franco-Canadien de Combinatoire, Montreal, North-Holland, New York (1980).

Bermond, J. C., and V. Faber, Decomposition of $\mathbb{K}_{n}^{*}$ into $k$-circuits. $J$. Combinatorial Theory $B$ 21(1976), 146-155.

Bermond, J. C., A. Germa, M. C. Heydemann, and D. Sotteau, Chemins et circuits dans les graphes orientés. Proc. Coll. Franco-Canadien de Combinatoire, Montréal, North Holland, New York (1980). 
Bermond, J. C., A. Germa, M. C. Heydemann, and D. Sotteau, Longest paths in digraphs. Preprint (1980a).

Bermond, J. C., A. Germa, M. C. Heydemann, and D. Sotteau, Girth in digraphs, J. Graph Theory 4(1980b) 337-341.

Bermond, J. C., C. Huang, and D. Sotteau, Balanced cycle and circuit designs: even cases. Ars Combinatoria 5(1978), 293-318.

Bermond, J. C. and Y. Kodratoff, Une heuristique pour le calcul de l'indice de transitivité d'un tournoi. RAIRO Informatique Théorique 10(1976), 83-92.

Bermond, J. C. and L. Lovász, Problem 3, Recent advances in graph theory, Proc. Coll. Prague. Academia Prague (1975), 541.

Bermond, J. C., J. M. S. Simoes-Pereira, and C. Zamfirescu, Homogeneously traceable digraphs. Math. Japonica 4(1979), 423-426.

Bermond, J. C. and D. Sotteau, Graph decompositions and G-designs. Proc. 5th British Combinatorial Conf., Aberdeen, Congressus Numerantium XV. Utilitas Math. Publ. (1976), 53-72.

Bermond, J. C. and D. Sotteau, Cycle and circuit designs, odd case, Beiträge zur Graphentheorie und deres Anwendungen. Proc. Coll. Oberhof Illmenau (1978), 11-32.

Bondy, J. A., Pancyclic graphs I. J. Combinatorial Theory B 11(1971), 8084.

Bondy, J. A., Large cycles in graphs. Discrete Math. 1(1971 a), 121-132.

Bondy, J. A., Variations on the Hamiltonian theme. Canad. Math. Bull. 15(1972), 57-62.

Bondy, J. A., Pancyclic graphs. Proc. 2nd Louisiana Conf. on Combinatorics, Graph Theory and Computing. Utilitas Math. Publ. (1972a), 167-172.

Bondy, J. A., Diconnected orientations and a conjecture of Las Vergnas. $J$. London Math. Soc. 14(1976), 277-282.

Bondy, J. A., Hamilton cycles in graphs and digraphs. Proc. 9 th Southeastern Conf. on Combinatorics, Graph Theory and Computing, Boca Raton, Congressus Numerantium XXI. Utilitas Math. Publ. (1978), 328.

Bondy, J. A. and M. Simonovits, Cycles of even lengths in graphs. $J$. Combinatorial Theory $\mathbb{B}$ 16(1974), 97-105.

Bondy., J. A. and C. Thomassen, A short proof of Meyniel's theorem. Discrete Math. 19(1977), 195-197.

Caccetta, L. and R. Häggkvist, On minimal digraphs with given girth. Proc. 8th Southeastern Conf. on Combinatorics, Graph Theory and Computing, Boca Raton, Utilitas Math. Publ., Congressus Numerantium XXI (1978), 181-187.

Camion, $\mathrm{P}$., Chemins et circuits hamiltoniens des graphes complets. $C . R$. Acad. Sci. Paris 249(1959), 2151-2152.

Camion, $P_{\circ}$, Une propriété des circuits hamiltoniens des graphes complets antisymetriques. Cahiers CERO, Bruxelles 15(1973), 225-228. 
Chartrand, G., D. Geller, and S. Hedetniemi, Graphs with forbidden subgraphs, J. Combinatorial Theory B 10(1971), 12-41.

Chartrand, G. and H. V. Kronk, Randomly traceable graphs. Siam J. Appl. Math. 16(1968), 696-700.

Chartrand, G., H. V. Kronk, and D. R. Lick, Randomly Hamiltonian digraphs. Fund. Math. 65(1969), 223-226.

Christofides, N. The travelling salesman problem, a survey. Preprint (1980).

Chvátal, V., On Hamilton's ideals. J. Combinatorial Theory B 12(1972), 163-168.

Chvátal, V. and P. Erdös, A note on Hamiltonian circuits. Discrete Math. 2(1972), 111-113.

Chvátal, V. and C. Thomassen, Distances in orientations of graphs. $J$. Combinatorial Theory B 24(1978), 61-75.

Corradi, K. and A. Hajnal, On the maximal number of independent circuits of a graph. Acta Math. Acad. Sci. Hungar. 14(1963), 423-443.

Dirac, G. A., Some theorems on abstract graphs. Proc. London Math. Soc. 2(1952), 69-81.

Dirac, G. A., Note on Hamilton circuits and Hamilton paths. Math. Ann. 206(1973), 139-147.

Dirac, G. A. and C. Thomassen, Graphs in which every finite path is contained in a circuit. Math. Ann. 203(1973), 65-75.

Douglas, R. J., Tournaments that admit exactly one Hamiltonian circuit, Proc. London Math. Soc. 21(1970), 716-730.

Egorycev, G. P., The method of coefficients and a generating function for the number of nonisomorphic tournaments that have a unique Hamiltonian contour (Russian). Kombin. Anal. 3(1974), 5-9.

Erdös, P., On the structure of linear graphs, Israel J. Math.1(1963), 156160.

Erdös, P. and T. Gallai, On maximal paths and circuits of graphs. Acta Math. Acad. Sci. Hung. 10(1959), 337-356.

Erdös, P. and J. W. Moon, On the sets of consistent arcs in a tournament. Canad. Math. Bull. 8(1965), 269-271.

Erdös, P. and L. Pósa, On the maximal number of disjoint circuits in a graph. Publ. Math. Debrecen 9(1962), 3-12.

Erdös, P. and L. Pósa, On independent circuits contained in a graph. Canad. J. Math. 17(1965), 347-352.

Erdös, P. and W. T. Trotter, When the Cartesian product of directed cycles is Hamiltonian. J. Graph Theory 2(1978), 137-142.

Forcade, R. Parity of paths and circuits in tournaments. Discrete Math. 6(1973), 115-118.

Fortune, S., J. Hopcroft, and J. Wyllie, The directed subgraph homeo-

- morphism problem. Theor: Comput. Sci. 10(1980), 111-121.

Fouquet, J. L., Problemes hamiltoniens dans les puissances de graphes, Problemes Combinatoires et Théorie des Graphes. Proc. Coll. Orsay. CNRS Publ. (1978), 145-147. 
Fouquet, J. L. and J. L. Jolivet, Graphes hypohamiltoniens orientés, Problemes Combinatoires et Théorie des Graphes. Proc. Coll. Orsay. CNRS Publ. City (1978), 149-151.

Frank, A., Problem at the Int. Coll. on Algebraic Methods in Graph Theory, Szeged (1978).

Gallai, $T_{3}$ On directed paths and circuits, Theory of Graphs. Proc. Colloq. Tihany 1966, Academic Press, New York (1968), 115-118.

Gallai, T., Problem 6 in: Theory of Graphs, Proc. Colloq. Tihany 1966, Academic Press, New York (1968a), 362.

Garey, M., On enumerating tournaments that admit exactly one Hamiltonian circuit. J. Combinatorial Theory $\mathbb{B}$ 13(1972), 266-269.

Ghouila-Houri, A., Une condition sufficante d'existence d'un circuit Hamiltonian. C. R. Acad. Sci. Paris 25(1960), 495-497.

Ghouila-Houri, A., Flots et tensions dans un graphe. Ann. Scient. Ac. Norm. Sup. 81(1964); 317-327.

Goldberg, M. and J. W. Moon, Arc mappings and tournament isomorphisms. J. London Math. Soc. 3(1971), 378-384.

Goldberg, M. and J. W. Moon, Cycles in k-strong tournaments. Pacific J. Math. 40(1972), 89-96.

Grant, D., F. Jaeger, and C. Payan, On graphs without antidirected elementary cycles and related topics. Preprint (1979).

Grötschel, M. and F. Harary, The graphs for which all strong orientations are Hamiltonian. J. Graph Theory 3(1979), 221-224.

Grötschel, M., and Y. Wakabayashi, Hypohamiltonian digraphs, Report 7891-OR, Universität Bonn (1978).

Grötschel, M., C. Thomassen, and Y. Wakabayashi, Hypotraceable digraphs. J. Graph Theory (1980).

Grünbaum, B., Exercise 16.26, in $\mathbb{F}$. Harary, Graph Theory. AddisonWesley, Reading, Mass. (1969), 211.

Grünbaum, B., Problem 31 in Combinatorial structures and their applications, Proc. Coll. Calgary. Gordon and Breach Science City (1969a), 504-505.

Grünbaum, B., Antidirected Hamiltonian paths in tournaments, J. Combinatorial Theory $\mathbb{1}(1971), 249-257$.

Gyori, E., Strongly connected digraphs with few cycles. Preprint Math. Inst. HHungar. Acad. Sci. 26(1978).

Hakimi, S. L. and E. F. Schmeichel, Pancyclic graphs and a conjecture of Bondy and Chvátal. J. Combinatorial Theory B 17(1974), 22-34.

Hamidoune, Y., Sur les parcours hamiltoniens dans les graphes orientés. Discrete Math. 26(1979), 227-234.

Hamidoune, Y., Connectivity of transitive digraphs and an application to combinatorial group theory. Proc. Coll. France-Canad. de Combinatoire, Montreal, Annals of Discrete Math. (1980).

Hamidoune, Y., A note on the girth of a digraph. Preprint (1980a).

Häggkvist, R., On the number of edge disjoint circuits in graphs. Preprint, Aarhus Universitet (1975). 
Häggkvist, R., On F-Hamiltonian graphs. Preprint, University of Umeaa (1977).

Häggkvist, R., Problem in Problem Session of the 6th British Combinatorial Conf., London, July (1977a) (unpublished).

Häggkvist, R. and C. Thomassen, On pancyclic digraphs. J. Combinatorial Theory $B$ 20(1976), 20-40.

Harary, F. and L. Moser, The theory of round robin tournaments. Amer. Math. Month. 73(1966), 231-246.

Harary, F., R. Z. Norman, and D. Cartwright, Structural models, An Introduction to the Theory of Directed Graphs. Wiley, New York (1965).

Harary, F. and E. Palmer, Graphical Enumeration. Academic Press, New York (1973).

Heydemann, M. C., On cycles and paths in digraphs. Discrete Math. 31(1980), 217-219.

Heydemann, M. C., Thesis, Université Paris-Sud (1980a).

Jackson, B., Hamilton cycles in regular 2-connected graphs. J. Combinatorial Theory B 29(1980), 27-46.

Jackson, B., Decompositions of graphs into cycles. Preprint (1980a).

Jackson, B., Paths and cycles in oriented graphs. Proc. Coll. Franco-Canad. de Combinatoire, Montreal, Annals of Discrete Math. (1980b).

Jackson, B., Long paths and cycles in oriented graphs. J. Graph Theory (1981). To appear.

Jakobsen, O. S., Cycles and paths in tournaments. Thesis, University of Aarhus (1972).

Johnson, D. B., Finding all elementary circuits of a directed graph. SIAMJ. Comput. 4(1975), 77-84.

Jolivet, J. L., Hamiltonian pseudo cycles in graphs. Proc. 5th Southeastern Conf. on Combinatorics, Graph Theory and Computing, Congressus Numerantium X, Utilitas Math. (1974), 529-533.

Jung, H. A., On subgraphs without cycles in a tournament, Comb. Theory and its Appl., Proc. Colloq. Balatonfüred 1969. North-Holland 2(1970), 675-677.

Kasteleyn, P. W., A Soluble self avoiding problem. Physica 29(1963), 1319-1337.

Kendall, M. G. and B. Babington Smith, On the method of paired comparisons. Biometrika 33(1940), 239-251.

Koh, K. M., Even circuits in directed graphs and Lovász' conjecture. Bull. Malaysian Math. Soc. 7(1976), 47-52.

Komlós, J. and E. Szemerédi, Limit distribution of the existence of Hamilton cycles in a random graph. Preprint (1976).

Koršunov, A. D., Solution of a problem of Erdös and Rényi on Hamiltonian cycles in nonoriented graphs. Soviet Math. Dokl. 19(1976), 760-764.

Korvin, G., Some combinatorial problems on complete directed graphs, Theory of graphs. Proc. International Symposium Rome 1966. Dunod, Paris, and Gordon and Breach, New York (1967), 197-202. 
Kosaraju, S. R., On independent circuits of a digraph. J. Graph Theory 1(1977), 379-382.

Kotzig, A., The decomposition of a directed graph into quadratic factors consisting of cycles, Acta F. R. N. Univ. Comment. Math. XXII (1969), 27-29.

Kotzig, A., On the maximal order of cyclicity of antisymmetric directed graphs. Discrete Math. 12(1975), 17-25.

Las Vergnas, M., Sur une propriétés des arbres maximaux dans un graphe. C. R. Acad. Sci. Paris 272(1971), 1297-1300.

Las Vergnas, M., Sur les circuits dans les sommes complétées de graphes orientés. Cahiers du CERO, Bruxelles 15(1973), 231-24.4.

Las Vergnas, M., Sur le nombre de circuit dans un tournoi fortement connexe. Cahiers du CERO, Bruxelles 17(1975), 261-265.

Las Vergnas, M., Problem in: Problem Session, Proc. 5th British Conf. Congressus Numerantium XV. Utilitas Math. Publ. (1976), 689.

Lehel, J., Counterexample for Häggkvist's conjecture. Preprint (1980).

Lempel, A., On extremal factors of the de Bruijn Graphs. J. Combinatorial Theory B 11(1971), 17-27.

Lesniak-Foster, L., Some recent results in Hamiltonian graphs. J. Graph Theory 1(1977), 27-36.

Lewin, M., On maximal circuits in directed graphs. J. Combinatorial Theory B 18(1975), 175-179.

Lovász, L., Problem 11 in: Combinatorial Structures and theirApplications. Proc. Coll. Calgary. Gordon and Breach, New York (1970), 497.

Lovász, $\mathbb{L}$., On two minimax theorems in graph. J. Combinatorial Theory $B$ 21(1976), 96-103.

Lucchesi, C. L. and D. H. Younger, A minimax theorem for directed graphs. J. London Math. Soc. 17(1978), 369-374.

Marcus, D. A., Spanning subgraphs of $k$-connected digraphs. Preprint (1979).

Mateti, P. and N. Deo, On algorithms for enumerating all circuits of a graph. SIAM J. Comput. 5(1976), 90-99.

Meyniel, $\mathrm{H}$., Une condition suffisante d'existence d'un circuit hamiltonien dans un graphe orienté, J. Combinatorial Theory B 14(1973), 137147.

Meyniel, H., Extension des théorèmes de Turán et de Brooks et notion de stabilité et de coloration dans les graphes orientés. Preprint (1980).

Minoux, M., Démonstration constructive du Théoreme de Meyniel: un algorithme en $O\left(n^{4}\right)$ pour la détermination d'un circuit hamiltonien. Preprint (1980).

Moon, J. W., On the number of edge-disjoint cycles in graphs. Canad. Math. - Bull. 7(1964), 519-523.

Moon, J. W., The minimum number of spanning paths in a strong tournament. Publ. Math. Debrecen 13(1966), 145-168. 
Moon, J. W., Topics on Tournaments. Holt, Rinehart, and Winston, New York (1968).

Moon, J. W. and L. Moser, On the distribution of 4-cycles in random bipartite tournaments. Canad. Math. Bull. 5(1962), 5-12.

Müller, V. and J. Pelant, The number of Hamiltonian circuits. J. Combinatorial Theory B 24(1978), 223-227.

Nash-Williams, C. St. J. A., Problem 47 in: Theory of Graphs, Proc. Colloq. Tihany 1966. Academic Press, New York (1968), 366.

Nash-Williams, C. St. J. A., Hamilton circuits in graphs and digraphs, The many facets of graph theory. Springer Verlag Lecture Notes 110(1969), 237-243.

Nash-Williams, C. St. J. A., Valency sequences which force graphs to have Hamiltonian circuits: Interim Report. University of Waterloo (1970).

Nash-Williams, C. St. J. A., Hamiltonian circuits, Studies in Graph Theory Part II. Studies in Mathematics 12, M. A. A., Washington 1975, 301360.

Ninćak, J., On a conjecture by Nash-Williams. Comment. Math. Univ. Carolinae 14(1973), 135-138.

Ore, O., Note on Hamilton circuits, Amer. Wath. Monthly 67(1960), 55.

Ore, O., Arc coverings of graphs. Ann. Math. Pure Appl. 55(1961), 315321.

Overbeck-Larisch, M., Hamiltonian paths in oriented graphs. J. Combinatorial Theory B 21(1976), 76-80.

Overbeck-Larisch, M., A theorem on pancyclic oriented graphs. J. Combinatorial Theory $B$ 23(1977), 168-173.

Palesti, $\mathbb{I}_{\text {, }}$ On the threshold distribution function of cycles in a directed random graph. Studia Sci. Math. Hungar. 6(1971), 67-73.

Pierce, $A_{\text {. }} \mathbb{R}$. Bibliography on algorithms for shortest path, shortest spanning tree, and related circuit routing problems, 1956-1974, Networks $5(1975), 129-149$.

Pósa, L., A theorem concerning Hamiltonian lines. Publ. Math. Inst. Hungar. Acad. Sci. 7(1962), 225-226.

Pósa, L., On the circuits of finite graphs. Publ. Math. Inst. Hungar. Acad. Sci. 8(1963), 355-361.

Pósa, L., Hamiltonian circuits in random graphs. Discrete Math. 14(1976), 359-364.

$\operatorname{Read}, \mathbb{R}$. C. and $\mathbb{R} . \mathbb{E}$. Tarjan, Bounds on backtrack algorithms for listing cycles, paths, and spanning trees. Networks 5(1975), 237-252.

Rédei, L., Ein kombinatorischer Satz. Acta Litt. Szeged 7(1934), 39-43.

Reid, K. B., $l$-cycles in tournaments having no $k$-cycles. Proc. $2 n d$ Louisiana Conf. on Combinatorics, Graph Theory, and Computing, Congressus Numerantium III. Univ. of Manitoba (1971), 473-482.

Rosenfeld, M., Antidirected Hamiltonian circuits in tournaments. J. Combinatorial Theory $\mathbb{B}$ 16(1974), 234-242. 
Roy, M., Nombre chromatique et plus long chemins d'un graphe. $R F A I R O$ 1(1967), 127-132.

Sekanina, M. On an ordering of the set of vertices of a connected graph. Publ. Fac. Sci. Univ. Brno 412(1960), 137-142.

Sheehan, J., The multiplicity of Hamiltonian circuits in a graph. Recent Advances in Graph Theory, Proc. Prague Symp., Akad Prague (1975), 447-480.

Skupién, Z. and A. P. Wojda, On highly Hamiltonian graphs, Bull. Acad. Sci. 22(1974), 463-471.

Sotteau, D., Decompositions of $K_{m, n}\left(K_{m, n}^{*}\right)$ into cycles (circuits) of length $2 k$. J. Combinatorial Theory $B$ 29(1980).

Sotteau, D., Thesis, Université Paris-Sud (1980a).

Szele, T., Kombinatorische Untersuchungen über gerichtete vollständige Graphen. Publ. Math. Debrecen 13(1966), 145-168.

Szwarcfiter, I. L. and P. E. Lauer, Finding the elementary cycles of a directed graph in $O(n+m)$ per cycle. Univ. of Newcastle upon Tyne (1974).

Thomassen, C., On randomly Hamiltonian graphs. Math. Ann. 200(1973), 195-208.

Thomassen, C., Antidirected Hamiltonian circuits and paths in tournaments. Math. Ann. 201(1973a), 231-238.

Thomassen, C., Graphs in which every path is contained in a Hamiltonian path. J. Reine Angew. Math. 268/269 (1974), 271-282.

Thomassen, C., Transversals in the lexicographic product of directed graphs. Math. Sci. Humaines. 51(1975), 43-45.

Thomassen, C., Paths and cycles in graphs. Ph.D. Thesis, University of Waterloo (1976).

Thomassen, C., An Ore-type condition implying a digraph to be pancyclic. Discrete Math. 19(1977), 85-92.

Thomassen, C., Hypohamiltonian graphs and digraphs. Theory and applications of graph. Springer Verlag Lecture Notes 642(1978), 557-570.

Thomassen, C., Long cycles in digraphs with constraints on degrees, Survey in Combinatorics. Proc. 7th British Combinatorial Conf., London Math. Soc. Lecture Notes 38, Cambridge University Press (1979), 211-228.

Thomassen, C., Hamiltonian connected toumaments. J. Combinatorial Theory $B$ 28(1980a), 142-163.

Thomassen, C., On the number of Hamiltonian cycles in tournaments. Discrete Math. 31(1980b), 315-323.

Thomassen, C., Edge-disjoint Hamiltonian paths and cycles in tournaments. Preprint, Aarhus Universitet (1980c).

Thomassen, C., Long cycles in digraphs. Proc. London Math. Soc. (1981).

Tillson, T., A Hamiltonian decomposition of $K_{2 m}^{*}, 2 m \geq 8$. J. Combinatorial Theory $B$ 29(1980), 68-74. 
Tutte, W. T., A theorem on planar graphs. Trans. Amer. Math. Soc. 82(1956), 99-116.

Waldrop, C., Ph.D. Thesis, Louisiana State University (1978).

Walther, H. and H. J. Voss, Über Kreise in Graphen. VEB Deutscher Verlag 1974.

Whitney, H., Congruent graphs and the connectivity of graphs. Amer. J. Math. 54(1932), 150-168.

Woodall, D. R., Sufficient conditions for circuits in graphs. Proc. London Math. Soc. 24(1972), 739-755.

Woodall, D. R., Maximal circuits of graphs I. Acta Math. Acad. Sci. Hungar. 28(1976), 77-80.

Wright, E. M., For how many edges is a digraph almost certainly Hamiltonian. Proc. Amer. Math. Soc. 41(1973), 384-388.

Younger, D. H., Graphs with interlinked directed circuits. Proc. Midwest Symp. on Circuit Theory 2(1973), XVI 2.1-XVI 2.7. 